\title{
Les échanges de bovins vivants et de viande bovine dans le monde et dans l'UE : trajectoires productives et commerciales des principaux pays impliqués
}

V. CHATELLIER

INRA, Agrocampus Ouest, UMR SMART-LERECO, 44300, Nantes, France

Courriel : vincent.chatellier@inra.fr

Face à l'augmentation de la consommation mondiale de viande bovine et aux difficultés productives rencontrées dans de nombreux pays, les échanges internationaux de viande bovine se développent, avec l'arrivée de nouveaux acteurs à l'export tels que l'Inde. Si les flux de bovins vivants et de viande bovine sont importants entre ses États membres, l'UE demeure un acteur modeste du marché mondial, tant à l'import qu'à l'export.

La viande bovine est intégrée dans le régime alimentaire d'une part importante de la population mondiale. En 2015, la consommation moyenne annuelle de viande bovine s'élève à environ $10 \mathrm{~kg}$ en équivalent carcasse par habitant (FAO 2016), soit un peu moins de $7 \mathrm{~kg}$ de muscle (ou viande nette sans os). Ce niveau de consommation varie fortement selon les zones géographiques : il dépasse $35 \mathrm{~kg}$ dans plusieurs pays du continent américain, avoisine $16 \mathrm{~kg}$ dans l'Union Européenne (UE) et ne dépasse pas $2 \mathrm{~kg}$ en Inde (FranceAgriMer 2011a). L'augmentation de la demande mondiale de viande bovine tient surtout à l'essor démographique (croissance annuelle de la population mondiale de l'ordre de 1,1\% par an). Un recul de consommation est observé depuis environ deux décennies dans de nombreux pays développés, dont en France. La viande de volaille connaît, quant à elle, une croissance de consommation plus soutenue en raison d'un coût de production plus avantageux (meilleur indice de consommation et cycle de production plus court), d'une bonne image santé, d'une praticité d'utilisation et d'une moindre exposition aux interdits religieux (Henchion et al 2014).

D'après l'organisation des nations unies pour l'alimentation et l'agriculture (FAO 2016), la production mondiale de viande bovine s'élève, en 2015, à 67,9 millions de tonnes en équivalent carcasse (tec), soit un volume très inférieur à celui de la viande de volaille (114,9 millions de tec) et de la viande porcine (117,2 millions de tec). La production de viande bovine est fortement concentrée dans la mesure où les quatre premiers producteurs, à savoir les États-Unis (10,8 millions de tec en 2015), le Brésil (8,8 millions de tec), l'UE-28 (7,7 millions de tec) et la Chine (7 millions de tec), réalisent la moitié de la production mondiale. Les pays qui arrivent ensuite sont l'Inde, l'Argentine, l'Australie, le Mexique, la Russie et le Canada.

Pour faire face à la croissance de la demande mondiale de viande bovine, un développement organisé de la production a été mis en œuvre, parallèlement à l'adoption de techniques agricoles modernes (rationnement des bovins, sélection génétique, gestion sanitaire des troupeaux...). La production mondiale de viande bovine résulte aujourd'hui de bovins aux caractéristiques très hétérogènes tant en termes de races (des races laitières versus des races à viande, des races précoces versus des races tardives), que d'âge des animaux abattus (des veaux de boucherie aux bovins adultes de plus de dix ans) ou de poids. La production de viande bovine recouvre au moins trois grands types de modèles productifs (Kahn et Cottle 2014). Le premier, le plus historique, regroupe les bovins pour lesquels la production de viande n'est finalement que le sous-produit d'une activité principale, à savoir par exemple la production de lait, dont les besoins mondiaux ne cessent de croître. Dans certains pays en développement où la mécanisation est encore loin d'être généralisée, les bovins ont aussi parfois pour vocation première de servir de traction animale. Le deuxième type de modèle rassemble des bovins élevés de manière extensive sur de grands espaces et nourris essentiellement d'herbe via le pâturage. Bénéficiant de cycles de production souvent longs, avec une croissance lente des animaux, ces systèmes mobilisent peu d'intrants et sont, du moins dans certains pays, plutôt compétitifs au niveau des coûts de production. Le troisième type, le plus récent, regroupe des bovins engraissés de manière plus intensive. Les bovins bénéficient d'une alimentation basée sur de l'herbe cultivée, des céréales secondaires, des concentrés protéiques (dont les tourteaux de soja), voire parfois des sous-produits issus des industries agro-alimentaires. L'objectif est de favoriser une croissance rapide des bovins, dans des ateliers qui peuvent atteindre des tailles conséquentes comme c'est souvent le cas en Amérique du nord.

L'objectif de cet article est de proposer une analyse centrée sur l'évolution, au cours des quinze dernières années (20002015), du commerce international et européen dans le secteur bovin. Une distinction est faite entre le commerce de bovins vivants et celui de viande bovine, étudié ici plus finement. Cet article est structuré en trois parties. Après le rappel de quelques faits sur les échanges mondiaux dans le secteur bovin, la première partie s'intéresse à la situation des quatre principaux pays exportateurs, à savoir l'Australie, l'Inde, le Brésil et les ÉtatsUnis. La deuxième partie traite de la situation des principaux pays importateurs, à savoir la Chine (en y incluant Hong-Kong, mais aussi le Vietnam, compte tenu de l'importance des flux entre ces trois zones), le Japon et la Russie. 
La troisième partie aborde l'évolution du commerce européen dans le secteur bovin, en s'intéressant d'une part aux échanges extra-UE de l'UE et de ses États membres et, d'autre part, aux principaux flux intra-UE.

\section{1 / Les principaux exporta- teurs mondiaux dans le sec- teur bovin}

Dans le secteur bovin, les échanges internationaux portent nettement plus, tant en tonnage qu'en valeur, sur la viande bovine que sur des bovins vivants (Institut de l'Elevage 2016). Les échanges internationaux (hors commerce intra-UE) de viande bovine portent, en 2015, sur 9,1 millions de tonnes équivalent carcasse (tec), soit environ $13 \%$ de la production mondiale. En progression sur longue période, mais en recul par rapport à 2014 (9,6 millions de tec), les exportations de viande bovine sont influencées par un ensemble de facteurs dont des questions d'ordre sanitaire (encadré 1), climatique (certains exportateurs sont sensibles à la sécheresse qui affecte les productions fourragères), économique (croissance dans les pays en développement) et monétaire (évolution des parités).

Les flux de bovins vivants, qui font l'objet d'une réglementation sanitaire rigoureuse, se font surtout entre pays voisins dans la mesure où il est techniquement difficile, mais aussi coûteux, d'assurer un transport d'animaux vivants sur de longues distances. En 2015, les principaux exportateurs de bovins vivants sont le Mexique (1,21 million de têtes), l'Australie $(1,11$ million de têtes $)$, le Canada (0,83 million de têtes), l'UE-28 (0,62 million de têtes), l'Uruguay $(0,22$ million de têtes) et le Brésil ( 0,21 million de têtes). Ce commerce recouvre une diversité de bovins qu'il est possible de regrouper schématiquement en trois catégories :

i) des bovins reproducteurs qui viennent conforter le cheptel d'un pays donné dans le but de développer la production intérieure et d'améliorer le potentiel génétique du troupeau national ; ces bovins étant commercialisés avec des valeurs unitaires plus élevés, ils peuvent parfois parcourir des distances plus importantes entre le pays d'origine et le lieu de destination;

ii) des bovins destinés à l'engraissement dans le pays acheteur. C'est la catégorie la plus importante en termes d'effectifs de bovins, à la lumière par exemple des exportations françaises de broutards à destination de l'Italie ;

Encadré 1. Le commerce de la viande bovine a été influencé par la fièvre aphteuse et l'ESB.

La fièvre aphteuse est une maladie virale grave qui affecte les artiodactyles, c'està-dire les animaux ayant des sabots fourchus, dont notamment les bovins. Hautement contagieuse, elle est endémique dans certaines parties de l'Asie et la majeure partie de l'Afrique et du Moyen-Orient. La majorité des pays d'Amérique du Sud ont appliqué des mesures de zonage et sont reconnus indemnes de fièvre aphteuse avec ou sans vaccination. La lutte contre cette maladie comporte des systèmes de détection et d'alerte précoces. Des mesures de contrôle et de surveillance strictes portant sur les importations et les déplacements transfrontaliers d'animaux permettent aussi de renforcer la protection des pays et des zones géographiques indemnes. Au fil des décennies, la fièvre aphteuse a eu une influence structurante sur les courants d'échanges de viande bovine entre les grandes zones du monde, en différenciant notamment la « zone Pacifique » (zone constituée de l'Amérique du Nord, de l'Asie du Sud-Est et de l'Océanie et exempte de la fièvre aphteuse sans vaccination) de la « zone Atlantique » (zone regroupant l'Amérique du Sud, l'Europe, le Proche et le Moyen-Orient, l'Asie du Sud et l'Afrique et pratiquant la vaccination contre la fièvre aphteuse, ce qui n'empêche d'ailleurs pas de fréquentes épizootie). II faut noter que I'UE a stoppé la vaccination contre la Fièvre aphteuse depuis le début des années 1990, et a connu une dernière épizootie importante en 2001-2002.

L'Encéphalopathie Spongiforme Bovine (ESB), ou maladie dite « de la vache folle ", a été reconnue pour la première fois en 1986 en Angleterre. Potentiellement transmissible à l'Homme, cette maladie est causée par l'accumulation d'une forme pathogène de protéines, le prion. Non seulement elle a connu de nombreux foyers de contamination (en Europe, en Amérique du Nord et au Japon), mais elle a remis en question le précédent clivage géographique instauré par la fièvre aphteuse en touchant à la fois les zones Pacifique et Atlantique. Au travers de l'application d'embargos sanitaires, elle a eu un impact important sur les flux internationaux de viande bovine. Si l'Australie est exempte de cette maladie, il n'en va pas de même aux Etats-Unis, pays particulièrement fragilisés.

iii) des bovins lourds destinés à l'abattage dans le pays client. Il s'agit souvent de pays déficitaires en viande bovine qui attachent une certaine importance à la question du rituel de l'abattage ou de pays ayant des infrastructures d'abattage efficiente.

En 2015, la viande bovine occupe le premier rang des viandes échangées en valeur, mais le deuxième en volume derrière la viande de volaille (12,3 millions de tec) et devant la viande porcine $(7,2$ millions de tec). Ainsi, et contrairement à d'autres produits agricoles plus internationalisés (soja, sucre, céréales, poudre de lait...), la viande bovine est un produit qui est d'abord très largement consommé dans le pays de production.

Les exportations de viande bovine sur le marché international résultent d'un nombre limité de pays (encadré 2). En 2015, les dix principaux exportateurs sont, en effet, à l'origine de $85 \%$ de l'ensemble des flux internationaux. Il s'agit, par ordre décroissant en volume, de l'Australie (1,87 million de tec), de l'Inde (1,70 million de tec), du Brésil (1,69 million de tec), des États-Unis $(1,11$ million de tec), de la Nouvelle-Zélande (631 000 tec), du Canada (399 000 tec), de l'Uruguay (376 000 tec), du Paraguay (374000 tec), de l'UE (240 000 tec) et du Mexique (207 000 tec). Un développement de l'analyse est proposé ci-après pour les quatre principaux fournisseurs du marché mondial (figure 1).

\section{1 / L'Australie}

Avec une surface agricole conséquente (412 millions d'hectares) et une population limitée (23 millions d'habitants), l'Australie est, et depuis fort longtemps, un pays qui compte dans le commerce international des produits agroalimentaires (Hogan et Morris 2010). Si l'agriculture ne contribue que pour $2 \%$ au produit intérieur brut et n'emploie que $3 \%$ des actifs, elle joue un rôle déterminant dans la balance commerciale du pays (Silva 2014). L'Australie compte un cheptel de bovins de 27,4 millions de têtes en 2015 , dont 12,5 millions de vaches allaitantes (et génisses) et 1,7 million de vaches laitières. Ce cheptel est concentré pour une grand part dans les trois États situés à l'est du pays, à savoir le Queensland (41\% du cheptel national), la Nouvelle-Galles du Sud $(20 \%)$ et Victoria $(15 \%)$. À l'Ouest, l'Australie occidentale ne compte que pour $9 \%$ du cheptel national. La production australienne de viande bovine atteint 2,5 millions de tec en 2015 , soit $4 \%$ du total mondial. Avec $13 \%$ de la production agricole finale, la viande 
Encadré 2. Sources statistiques et éléments méthodologiques.

Les données statistiques utilisées à l'échelle internationale pour discuter de la question des échanges sont celles issues de la base de données BACI développée par le CEPII (Centre de recherche français dans le domaine de l'économie internationale) à partir des données primaires de la base de données COMTRADE issue de la division statistique des nations Unies. La base $\mathrm{BACl}$ est construite selon une procédure originale qui concilie les déclarations de l'exportateur et de l'importateur. En permettant de disposer partout dans le monde d'un seul et même code de désignation pour une même marchandise (du moins au niveau dit "SH6 »), le Système Harmonisé $(\mathrm{SH})$ des douanes contribue à l'harmonisation des régimes douaniers, des procédures commerciales et douanières et facilite l'échange de données commerciales. Ce système harmonisé est utilisé par 195 pays et couvre plus de 95\% du commerce mondial. Dans le cas de l'Inde (et seulement dans ce cas), ce sont les données primaires de COMTRADE qui sont utilisées, avant l'application du traitement selon la méthode BACI.

Les données statistiques utilisées à l'échelle de l'UE et des États membres sont celles issues de la base de données COMTEXT. La Nomenclature Combinée (NC) définie au niveau européen est constituée de 8 chiffres, soit les 6 premiers du système harmonisé et de deux chiffres complémentaires. Elle détermine notamment les droits de douane à l'importation dans I'UE et permet l'élaboration des statistiques du commerce extérieur. Suite à l'adoption du marché unique le $1^{\text {er }}$ janvier 1993 , les formalités douanières entre les États membres ont été supprimées, mais un nouveau système de collecte de données auprès des opérateurs a été mis en place pour traiter la question du commerce intra-communautaire. Ce système pose cependant parfois certaines questions méthodologiques car la vitesse du renseignement des informations varie entre les États membres. Ainsi, il arrive que les exportations d'un pays « $A$ » vers le pays « $B$ » ne correspondent pas toujours, en temps réel, aux importations déclarées par le pays « $B$ » en provenance du pays « $A$ ».

Le terme « viande bovine » utilisé tout au long de cet article correspond à une définition précise et cohérente entre les données internationales et européennes. Sans entrer ici dans la liste détaillée des codes SH ou NC impliqués, il regroupe les viandes fraîches et réfrigérées (avec des déclinaisons possibles pour identifier les carcasses, les découpes, les découpes désossées, les abats comestibles), les viandes congelées (avec des déclinaisons similaires) mais aussi les produits transformés (préparations et les viandes salées, séchées et fumées).

Les échanges sont indiqués en euros courants et en Tonne Équivalent-Carcasse (TEC). Un raisonnement en TEC (qui diffère du calcul en viande nette sans os, critère parfois utilisé) permet d'agréger, d'une certaine manière, des viandes sous toutes leurs présentations. Pour connaître la quantité équivalente de viande sous une forme donnée, on y applique un coefficient propre à chaque produit. Le coefficient utilisé est celui utilisé par les services de FranceAgriMer. Dans le cas de COMTRADE, ce coefficient est, par exemple, de 1 pour les carasses, les demi-carcasses et les découpes. II est de 1,3 pour les abats et les viandes salées, séchées et fumées. La nomenclature de COMEXT étant plus fine, le niveau de précision de l'estimation est renforcé.

Figure 1. Les principaux exportateurs de viande bovine dans le monde (Milliards d'euros courants).

(Source : BACI d'après Comtrade/Traitement INRA, SMART-LERECO).

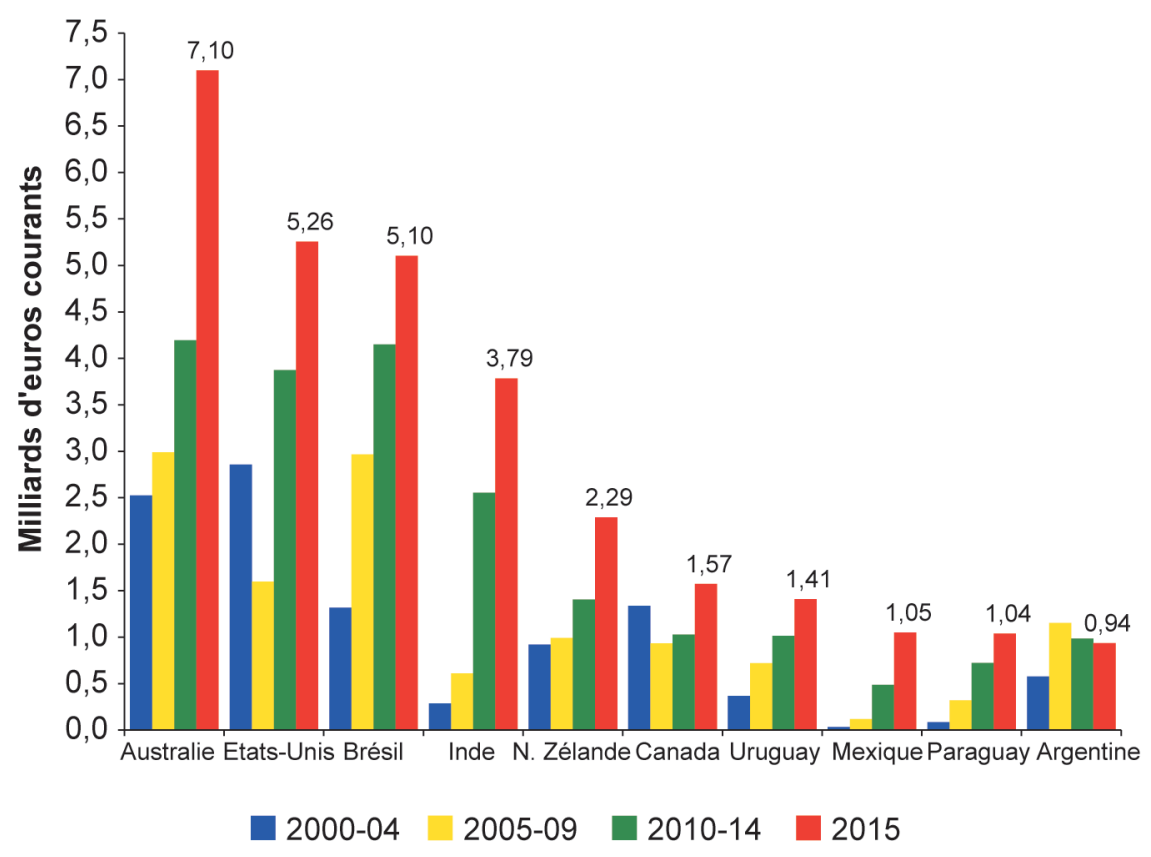

bovine est le deuxième produit agricole le plus important derrière le blé. Le potentiel de développement de l'élevage est cependant contraint par la faible part de problèmes d'accès à l'eau (sécheresse) et l'érosion des sols dans certaines régions (Bell et al 2011). terres arables (48 millions d'hectares), les
La production australienne de viande bovine relève essentiellement de deux types de systèmes productifs. Le premier, historique et compétitif au niveau des coûts de production, correspond à un système dit de «ranching » où les animaux sont nourris à l'herbe de façon extensive dans des paddocks à la taille souvent importante (plusieurs milliers d'hectares). Le second correspond au modèle, imaginé aux États-Unis, des "feed lots». Il s'agit d'élevages intensifs qui privilégient l'engraissement d'animaux maigres à partir notamment de céréales et de tourteaux dans des parcs situés à proximité souvent de zones céréalières. L'introduction des parcs d'engraissement en Australie remonte aux années 1950, période correspondant à une montée en puissance de la demande extérieure de viande bovine (Japon, Corée du Sud, Taiwan, États-Unis). Ces deux systèmes productifs sont complémentaires, l'un fournissant à l'autre des veaux sevrés et en bonne santé, l'autre libérant l'élevage extensif des bêtes à l'engraissement.

En dépit d'un niveau individuel de consommation de viande bovine élevé (environ $30 \mathrm{~kg}$ par habitant et par an), mais en raison d'un marché intérieur réduit, la consommation de viande bovine ne représente qu'une part limitée de la 
production intérieure (environ un quart). Il en résulte que l'Australie occupe, et depuis longtemps, une place importante dans le commerce mondial de bovins. Cette situation est renforcée par l'existence d'une situation sanitaire favorable. En effet, d'après l'Organisation mondiale de la santé animale (OIE), ce pays est indemne de fièvre aphteuse, comme cela est également le cas en Nouvelle-Zélande, en Amérique Centrale, en Amérique du Nord et en Europe Occidentale.

La position à l'export de l'Australie est également favorisée par son savoirfaire de filière. Ainsi, par exemple, ce pays a développé le "Meat Standard Australia » qui permet de prédire la qualité de la viande à partir de chaque combinaison " muscle/mode de cuisson ». Ce système, qui a été testé avec succès aux Etats-Unis, au Japon, en Corée du Sud et, plus récemment en Chine, stimule l'intérêt de la communauté scientifique étrangère, y compris française (Legrand et al 2013). La position commerciale de l'Australie est aussi confortée par ses politiques publiques. Membre du groupe de Cairns, l'Australie est un pays peu interventionniste en agriculture et un fervent partisan de la libéralisation des marchés agricoles dans le cadre des négociations multilatérales de l'Organisation Mondiale du Commerce -OMC- (Potard-Hay 2016). Pour dynamiser ses échanges, l'Australie a également signé de nombreux accords de libre-échange. Cela concerne le Closer Economic Relations Trade en 1983 avec la Nouvelle-Zélande ; les Free Trade Agreement avec Singapour (2003), la Thailande (2005), les Etats-Unis (2005), le Chili (2009) et la Malaisie (2013); l'accord avec la Nouvelle-Zélande et les pays signataires de l'ASEAN (Association des pays du sud est asiatiques en 2010). Depuis le 4 février 2016, l'Australie est également membre du «TransPacific Partnership Agreement » (TTP), un traité multilatéral de libre-échange qui vise à intégrer les économies des régions Asie-Pacifique et Amérique. Cet accord, récemment remis en cause par l'administration du nouveau Président américain (cf. infra), prévoit la suppression de près de 18000 droits de douanes, y compris sur les denrées agricoles, avec une diminution progressive dans le cas de la viande bovine.

L'Australie occupe le deuxième rang des pays exportateurs de bovins vivants $(1,11$ million de têtes) derrière le Mexique ; elle tient également le deuxième rang en valeur derrière le Canada et devant le Mexique. En valeur, les exportations australiennes de bovins vivants sont passées, en monnaie courante, de 308 millions d'euros en 2000 à 1,07 milliard d'euros en 2015. À cette dernière date, elles sont destinées principa-
Figure 2. Les exportations de l'Australie en viande bovine (Milliers de tec). (Source : BACI d'après Comtrade/Traitement INRA, SMART-LERECO).

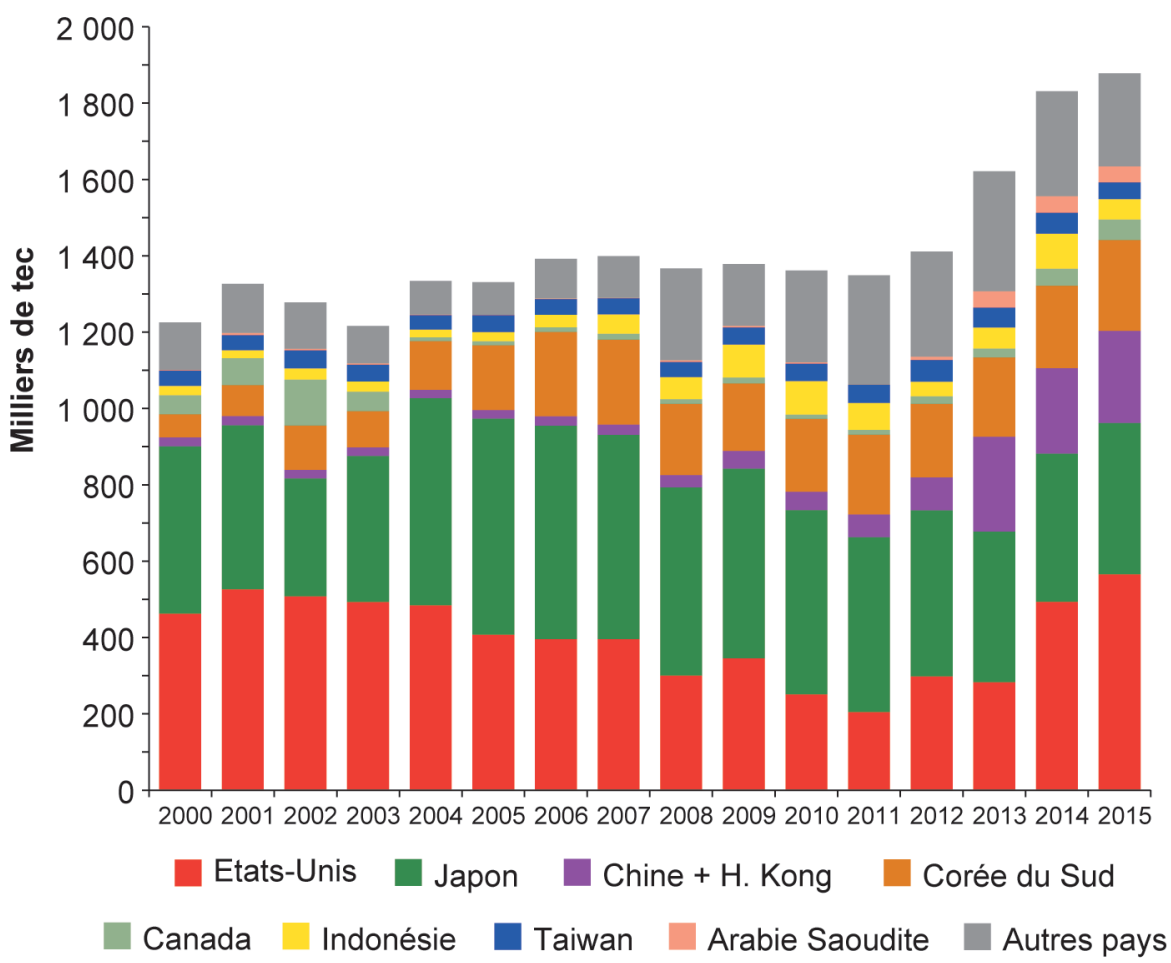

lement à l'Indonésie (35\% du total en valeur), le Vietnam $(28 \%)$, la Chine $(15 \%)$, la Russie (6\%), la Malaisie (4\%) et Israël (3\%). Si l’Égypte ouvre parfois ses portes aux bovins vivants en provenance d'Australie (34\% des exports en 2001), les flux sont très irréguliers d'une année à l'autre.

En 2015, l'Australie assure $20 \%$ des exportations mondiales (hors flux intraUE) de viande bovine en volume et elle se positionne en tête devant ses concurrents en valeur. Après avoir perdu sa place de leader au bénéfice du Brésil au milieu de la précédente décennie, ce pays occupe de nouveau le premier rang en 2015. Les exportations sont passées de 1,22 million de tec en 2000 à 1,87 million de tec en 2015, la hausse ayant surtout eu lieu au cours des cinq dernières années (figure 2). L'inflation accumulée tout au long de cette période et l'augmentation tendancielle du prix international de la viande bovine font que la hausse des exportations est plus nette lorsqu'elle est exprimée en valeur (de 2,37 à 7,09 milliards d'euros).

Au cours des années 2015-2016, les exportations de viande bovine d'Australie ont été d'une certaine manière confortées par la sécheresse qui sévissait dans le pays en ce sens que celle-ci a entraîné une décapitalisation de cheptel. Les exportations se font principalement sous la forme de viandes congelées $(61 \%$ des exportations en valeur) et de viandes fraîches désossées $(28 \%)$; les produits transformés occupent une place moins déterminante $(5 \%)$, de même que les viandes fraîches découpées $(3 \%)$ et en carcasse (1\%). En 2015, les exportations sont destinées principalement aux ÉtatsUnis (30\% des volumes), au Japon $(21 \%)$, à la Corée du Sud $(13 \%)$ et à la Chine (10\%). Viennent ensuite, mais plus loin derrière, le Canada, l'Indonésie, Taïwan et l'Arabie Saoudite. Exprimée en valeur, la part relative de ces pays est proche. Quelques précisions sont apportées ci-après pour les quatre principaux clients.

- Les exportations à destination des États-Unis se sont élevées à 565000 tec en 2015 (soit $37 \%$ du total des importations américaines), le tonnage le plus important réalisé depuis au moins quinze ans et l'un des flux les plus conséquents au monde (carte 1). Pour la première fois, les exportations australiennes devraient même dépasser la limite des contingents d'importation sur le marché américain. Après avoir baissé au profit du Japon et de la Corée du sud, les exportations vers les États-Unis ont été nettement relancées depuis 2011, le plus bas niveau de la période étudiée (204 000 tec). Cette relance est liée aux disponibilités internes à l'Australie, mais également à la baisse de la production américaine de viande bovine.

- Les exportations à destination du Japon (396000 tec en 2015) ont atteint leur niveau record en 2005 (566 000 tec). Suite à la signature, en 2014, d'un accord de partenariat économique entre ces deux pays, elles pourraient être relancées dans 
Carte 1. Les principaux courants d'échanges de viande bovine dans le monde en 2015 (milliers de tec). (Source : BACI d'après Comtrade/Traitement INRA, SMART-LERECO).

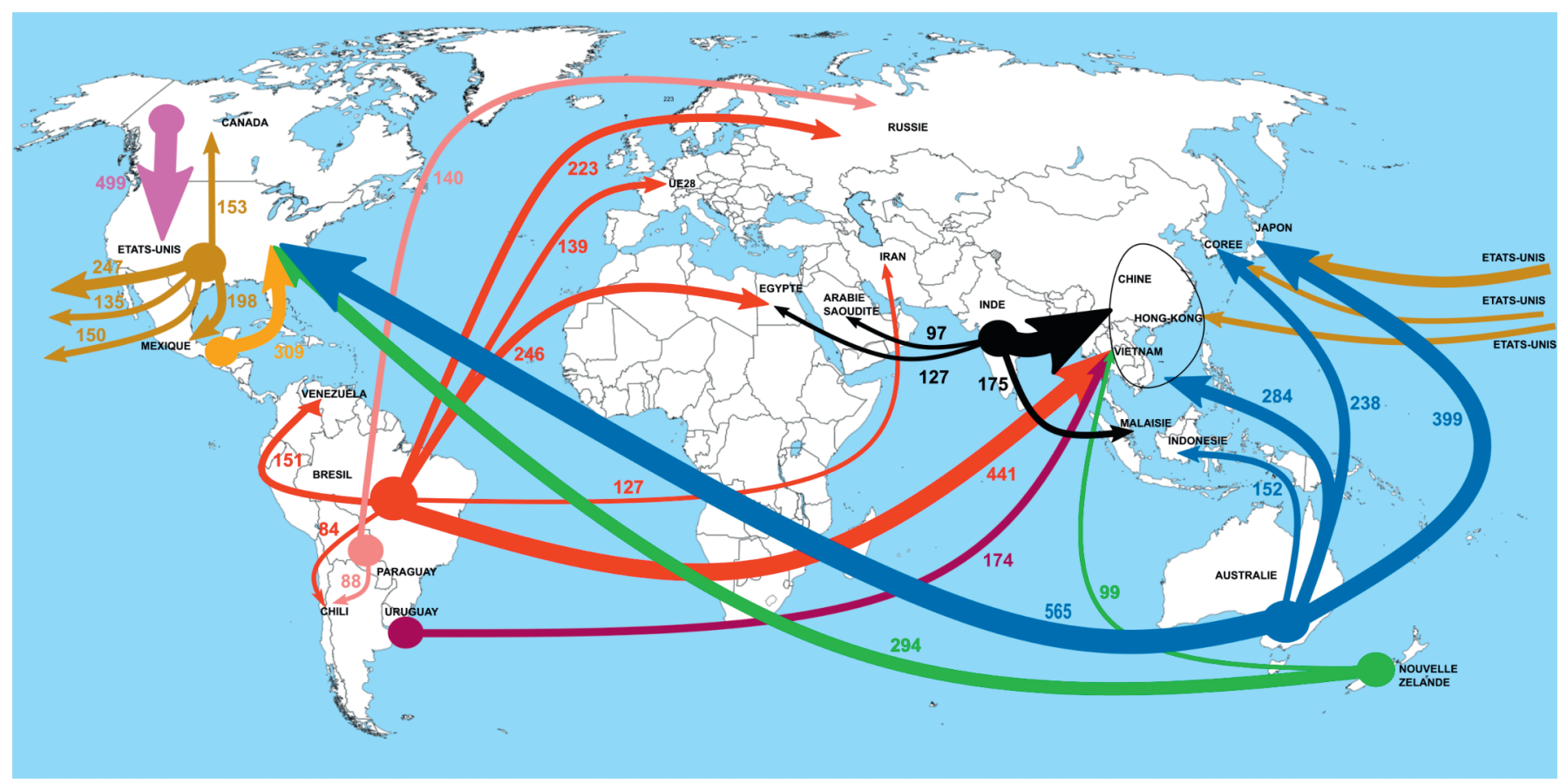

les prochaines années. En quinze ans, le tarif douanier de $38,5 \%$ appliqué sur la viande congelée et réfrigérée sera, en effet, progressivement réduit à respectivement 19,5 et $23,5 \%$ (USDA $2015-a$ ). En vertu de cet accord, le Japon a aussi réduit de $20 \%$ les droits sur les importations de bovins vivants en provenance d'Australie, sans restriction de volume. En dépit de ces accords, la baisse attendue de la population japonaise pourrait avoir un impact négatif sur ses besoins intérieurs.

- Les exportations à destination de la Corée du sud, pays structurellement déficitaire pour ce produit, se sont nettement développées pour atteindre 237000 tec en 2015, soit quatre fois plus qu'en 2000. L'Australie assure ainsi $58 \%$ des importations coréennes devant les États-Unis (33\%), la Nouvelle-Zélande (7\%), l'Uruguay $(3 \%)$ et le Canada $(3 \%)$.

- Les exportations à destination de la Chine atteignent 213700 tec en 2015 contre moins de 40000 tec tout au long de la période 2000-2011. Depuis 2012, « l'Empire du Milieu » offre de nouvelles opportunités de développement pour l'Australie (Edwards et al 2016).

Les importations de l'Australie en viande bovine sont marginales (15 000 tec en 2015, soit le plus haut niveau des vingt dernières années) relativement aux exportations et elles proviennent exclusivement de la Nouvelle-Zélande (9 500 tec) et des États-Unis (4 100 tec). Pour la Nouvelle-Zélande, pays géographiquement voisin qui a fortement développé sa production laitière au cours des quinze dernières années et est largement excédentaire en viande bovine $(+615000$ tec en 2015), l'Australie reste un client très modeste par rapport aux États-Unis (293000 tec) et à la Chine (96000 tec).

\section{2 / L'Inde}

Avec 1,3 milliard d'habitants en 2015 (dont $40 \%$ sont âgés de moins de 20 ans), l'Inde regroupe $18 \%$ de la population mondiale pour seulement $4 \%$ des terres agricoles (soit 180 millions d'hectares) et $4 \%$ des ressources en eau. L'agriculture se caractérise par d'innombrables exploitations de très petite taille (économie d'autosubsistance), une mécanisation peu développée, des rendements faibles et un développement limité des activités de transformation. L'Inde est le premier producteur mondial de lait (avec une forte croissance des besoins intérieurs) et le second pour le blé, le riz, le coton, le sucre, le thé ou les fruits et légumes. Si la part de l'agriculture dans le Produit Intérieur Brut (PIB) a fortement reculé (de $30 \%$ en 1990 à $17 \%$ en 2015), l'agriculture demeure le premier employeur du pays avec environ la moitié des actifs. $\mathrm{Ce}$ pays, qui applique des droits de douane élevés sur les produits agricoles importés et qui recourt à de nombreuses restrictions à l'importation, bénéficie d'un excédent commercial en croissance et positif dans le domaine agroalimentaire (16 milliards d'euros en 2014 selon les douanes indiennes). La part de l'agroalimentaire dans les exportations totales a considérablement baissé en passant de près de $40 \%$ au début des années soixantedix à seulement $10 \%$ aujourd'hui (Pouch et Kheraief 2016).
Dans le secteur bovin, l'Inde se distingue surtout par la très grande taille de son cheptel, le plus développé au monde. Il s'élève à près de 300 millions de têtes, dont $51 \%$ de zébus, $36 \%$ de buffles et $13 \%$ de croisements de zébus avec des races importées. En dépit de ce cheptel conséquent, mais en raison de la conformation des bovins et de leurs performances zootechniques, la production indienne de viande bovine (4,1 millions de tec en 2015 pour 38 millions de bovins abattus) ne représente que $6 \%$ de l'offre mondiale. En Inde, les bovins sont utilisés essentiellement pour la production laitière et pour la traction animale (cf. photo 1). L'augmentation soutenue de la production laitière influe positivement sur le développement du cheptel de femelles alors que l'essor de la mécanisation joue en défaveur de celui des mâles. Des rééquilibrages s'opèrent donc, au fil du temps, entre catégories de bovins.

La consommation individuelle de viande bovine en Inde (estimée à $1,7 \mathrm{~kg}$ par habitant et par an) est l'une des plus faibles au monde. Les habitudes alimentaires (le régime végétarien est adopté par près de $40 \%$ de la population), la grande pauvreté d'une part écrasante de la population et les interdits religieux sont les principaux facteurs qui influent sur cette situation. Les hindous $(80 \%$ de la population) considèrent que les femelles zébus (vaches) sont « sacrées » et, de fait, s'opposent à leur abattage et à leur consommation. La viande bovine issue des buffles et des zébus mâles n'est cependant pas sujette aux mêmes interdits religieux. De plus, les règles relatives à l'abattage (et au transport) des différentes 
Photo 1. Les bovins ont parfois une fonction de traction animale en Inde. Source : Vincent Chatellier.

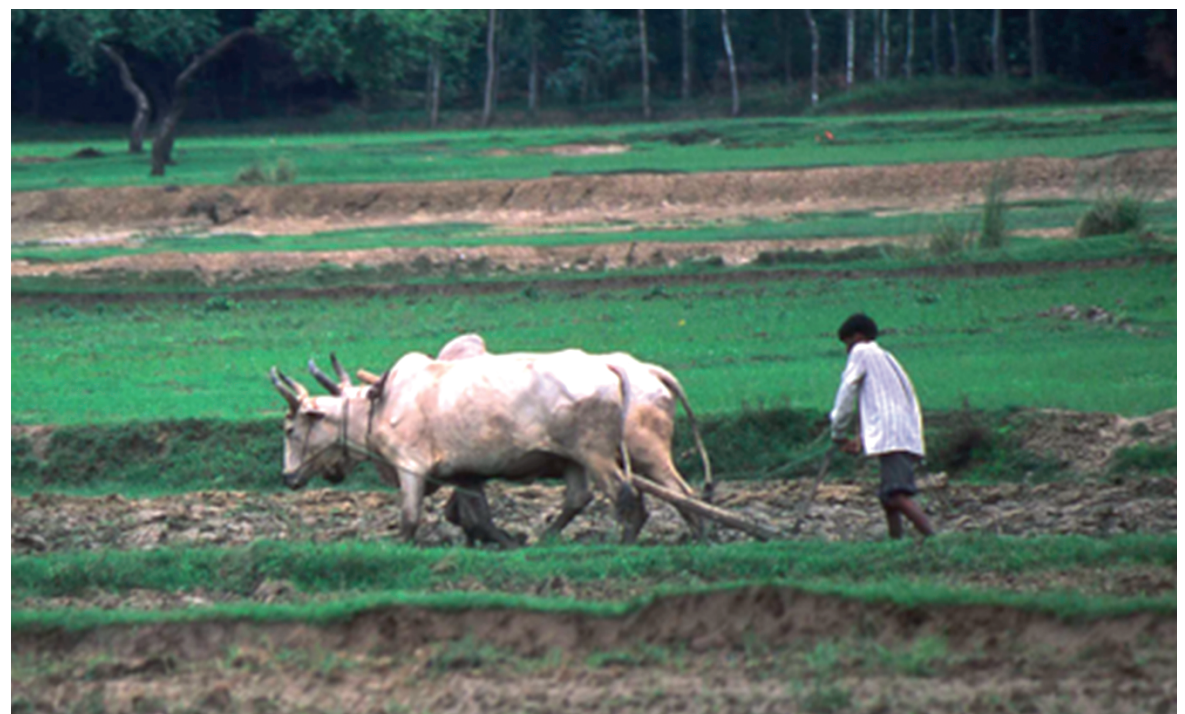

catégories de bovins sont complexes et déterminées au niveau de chaque État et non pas à l'échelle fédérale du pays (USDA, 2015b).

L'Inde, dont les importations sont nulles ou marginales dans le secteur bovin (y compris en animaux vivants), a fortement développé ses exportations de viande bovine au cours de la précédente décennie. Contrairement aux principaux autres pays étudiés pour lesquels les écarts d'estimation des exportations sont limités entre les différentes bases de données existantes sur le commerce, le cas de l'Inde est plus problématique. D'après les données de Comtrade (et sans l'application de la méthode BACI), les exportations de viande bovine de l'Inde sont passées de 266000 tec en 2000 (pour un montant de 272 millions d'euros) à 1,34 million de tec en 2015 (pour un montant de 3,79 milliards d'euros), après un pic atteint en 2014 (année où l'Inde aurait même détrôné l'Australie et le Brésil pour les exportations en volume). Ces exportations concernent surtout des bufflonnes laitières de réforme âgées de plus de dix ans et des buffles mâles utilisés pour la traction (avec un poids moyen de carcasse faible par rapport aux standards européens, c'est-àdire compris entre 140 et $200 \mathrm{~kg}$ ). Les bovins sont abattus selon le rite halal et la viande est exportée sous une forme désossée et congelée. Le développement rapide des exportations est imputable aux investissements réalisés par des sociétés familiales dans des outils industriels modernisés (Institut de l'Élevage 2015b). Les outils d'abattage agréés pour l'export sont localisés pour une part importante dans l'État de l'Uttar Pradesh qui assure à lui seul près de $70 \%$ des exportations indiennes de viande bovine. De même, trois groupes industriels (Allana, HMA et Hindagro) réalisent $60 \%$ des exportations du pays, lesquelles sont expédiées souvent par le port de Mumbai.

L'Inde n'étant pas reconnue « indemne de fièvre aphteuse » par l'Organisation Internationale de la santé animale (OIE), de nombreux pays développés (dont l'UE, les USA, le Japon, la Corée du sud) appliquent un embargo sur les viandes indiennes. La viande bovine indienne est donc commercialisée surtout dans des pays en développement de l'Asie du SudEst et du Moyen Orient où le prix d'achat constitue une variable cruciale. Le prix à l'export de la viande bovine indienne (environ 2,8 euros par kgec d'après Comtrade) est très compétitif en raison surtout du faible prix d'achat des bovins aux producteurs/détenteurs et du coût imbattable de la main-d'œuvre. Le premier pays client de l'Inde en viande bovine est, du moins au regard des seules informations disponibles dans les bases de données, le Vietnam, avec $43 \%$ du total des volumes exportés en 2015 . Cependant, et sans qu'il soit facile d'en connaître les volumes exacts, il est généralement admis qu'une très large part de ces importations sont destinées in fine au marché chinois. D'après certains auteurs (Landes et al 2016, Liu et Iqbal 2016), les exportations de l'Inde vers la Chine peuvent être approchées en considérant qu'elles correspondent à la différence entre les exportations indiennes officiellement annoncées au Vietnam et les importations officiellement annoncées du Vietnam en provenance de l'Inde. La Malaisie (10\% des volumes exportés) est le deuxième client de l'Inde, devant l'Égypte (8\%), l'Arabie Saoudite (5\%), la Thaillande (5\%), les Philippines (3\%), l'Irak $(3 \%)$ et l'Algérie (3\%).

\section{3 / Le Brésil}

Avec 200 millions d'habitants en 2015 et 275 millions d'hectares de surface agricole, le Brésil est l'un des tous premiers producteurs agricoles au monde et le troisième exportateur de biens agroalimentaires (Commission européenne, 2016). Le secteur agro-industriel au sens large (agriculture, industries agroalimentaires et agroéquipements) représente $22 \%$ du produit intérieur brut et $43 \%$ des exportations (Sabourin 2014). Si les exportations agroalimentaires brésiliennes (67 milliards d'euros en 2015) sont inférieures à celles de l'UE-28 (128 milliards d'euros) et des États-Unis (127 milliards d'euros), le solde agroalimentaire est bien meilleur (60 milliards d'euros). Les principaux clients du Brésil en agroalimentaire sont la Chine $(23 \%$ des exportations) et l'UE-28 (22\%). Le secteur agricole brésilien recouvre une grande diversité d'exploitations, avec, d'un côté, de grands domaines dédiés aux productions d'exportations et, de l'autre, de très nombreuses petites structures familiales centrées surtout sur le marché domestique. Au plan commercial, le Brésil est favorable à une libéralisation accrue des échanges agricoles et à une ouverture accentuée du marché européen dans le cadre de la négociation entre l'UE et plusieurs pays du Mercosur.

Le Brésil est le deuxième pays au monde, derrière l'Inde, pour la taille de son cheptel. Culminant à 100 millions de têtes en 1975, il a plus que doublé pour atteindre 210 millions de têtes dès 2005 (ABIEC 2016) ; il s'est ensuite stabilisé à ce niveau jusqu'à aujourd'hui. L'augmentation de la consommation intérieure de viande bovine, qui atteint un niveau près de quatre fois supérieur à la moyenne mondiale (soit $38 \mathrm{~kg}$ par habitant et par an), et l'essor de la demande internationale ont été les moteurs du développement du cheptel. Le troupeau brésilien est constitué principalement de races de type zébu, de la sous-espèce Bos indicus, reconnaissable à leur bosse sur le dos et à leurs oreilles tombantes. Les deux races de zébus les plus répandues sur le continent sud-américain sont la Nelore (race à viande) et, plus loin derrière, la Gir (race mixte lait/viande). Les autres bovins sont des races d'origine européenne de type Bos taurus, telles la Prim 'holstein, l'Angus ou la Hereford. Le cheptel brésilien est concentré pour près de $60 \%$ dans les six États suivants : Mato Grosso $(13,6 \%)$, Minas Gerais $(11,3 \%)$, Goiás $(10,3 \%)$, Mato Grosso do Sul $(10,1 \%)$, Pará $(8,9 \%)$ et Rio Grande do Sul $(6,7 \%)$.

Le Brésil est le deuxième producteur de viande bovine au monde dernière les États-Unis, avec 8,8 millions de tec en 2015 . Après un léger recul de la production au cours des dernières années, celleci pourrait de nouveau progresser dans les dix années à venir en raison des progrès de la génétique animale, de l'amé- 
lioration de la gestion des plantes fourragères (pour favoriser un niveau plus élevé de chargement) et des progrès en cours au niveau de l'efficacité alimentaire (FAO-OCDE 2016). Dans plusieurs régions, néanmoins, le développement des productions végétales (dont le soja, le maïs et la canne à sucre) entre en concurrence croissante avec les activités d'élevage. Ainsi, les surfaces de prairies, qui s'élèvent à 167 millions d'hectares à l'échelle nationale, ont reculé de près de $12 \%$ depuis 1990 . Les activités d'élevage donnent souvent lieu à une séparation marquée entre, d'une part, le naissage et l'engraissement et, d'autre part, la repousse et la finition (Institut de l'Élevage 2012). Elles relèvent majoritairement de systèmes d'élevage extensif à l'herbe, d'autant plus compétitifs que les apports alimentaires extérieurs sont limités et que les coûts en bâtiments sont modestes (Champion et Chotteau 2013). À côté de ces élevages extensifs, qui peuvent couvrir jusqu'à plusieurs milliers d'hectares, le Brésil a également mis en œuvre, à partir des années quatre-vingt, des feedlots ou confinamentos. Si les élevages intensifs de ce type se développent, ils ne couvrent aujourd'hui que 10 à $15 \%$ du total des bovins abattus au Brésil. Souvent situés dans des zones propices à la production des grains, ils sont dédiés à l'engraissement de broutards repoussés, pour une durée courte (trois mois).

Les exportations brésiliennes de viande bovine représentent, en 2015, 20\% de la production intérieure. Une part importante de la croissance de l'offre observée au cours des vingt dernières années a été mise au profit de la demande domestique. Pour les entreprises, l'accès aux marchés d'exportations est réglementé et dépend d'agréments sanitaires plus ou moins stricts. Pour exporter sur le marché international et commercialiser des viandes sur l'intégralité du territoire national, l'entreprise doit au préalable bénéficier d'un agrément délivré par le Service d'Inspection Fédéral. La moitié seulement de la production brésilienne de viande bovine est couverte par cet agrément. Les trois groupes agroalimentaires les plus puissants du secteur (JBS, Marfrig et Minerva) réalisent $56 \%$ des abattages nationaux et sont à l'origine de plus de $90 \%$ des exportations. L'entreprise JBS, concentre à elle seule, $34 \%$ des abattages et la moitié des exportations nationales de viande bovine. Cette forte concentration des acteurs industriels favorise la rationalisation des outils d'abattage et permet de générer des économies d'échelle qui constituent un atout face à la concurrence internationale (FranceAgriMer 2011b).

Au niveau du commerce de bovins vivants, les importations du Brésil sont
Figure 3. Les exportations du Brésil en viande bovine (Milliers de tec). (Source : BACI d'après Comtrade/Traitement INRA, SMART-LERECO).

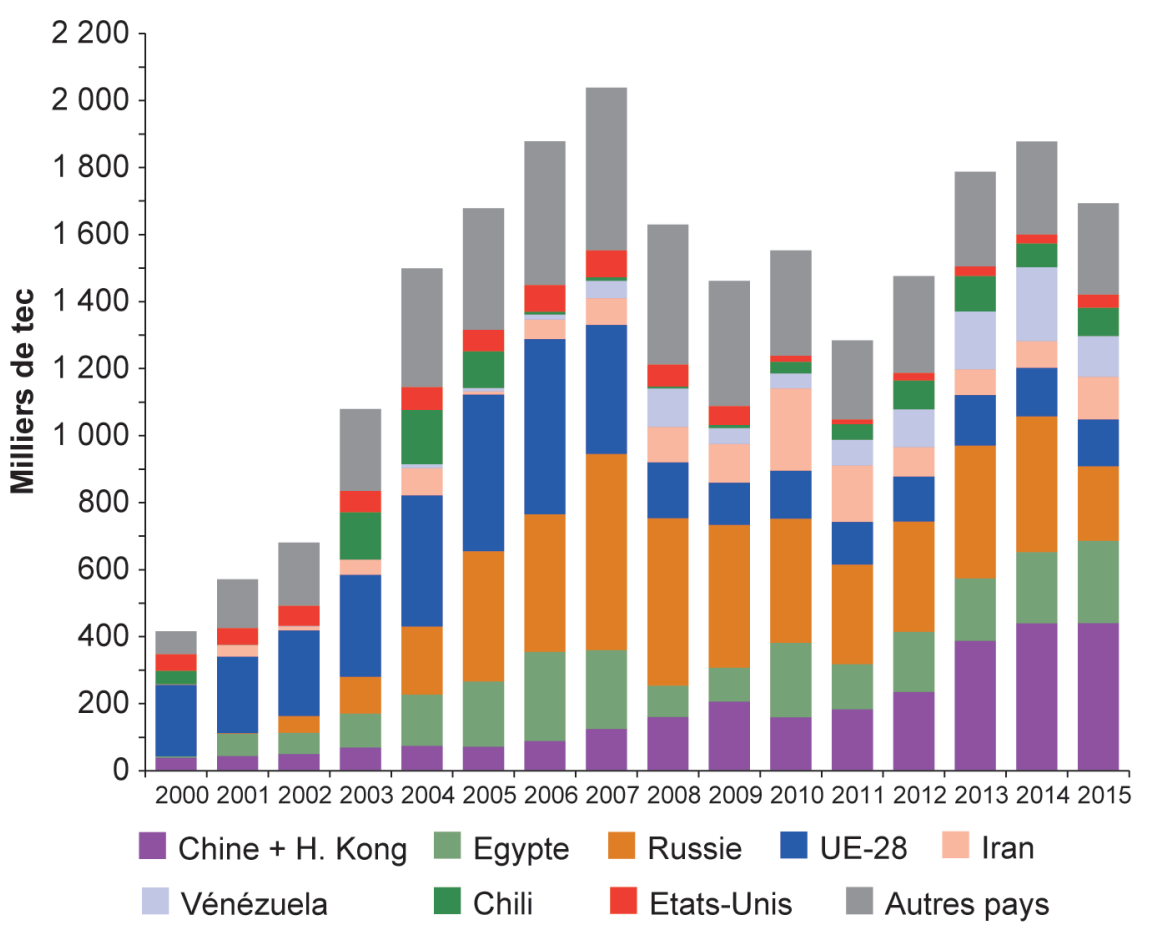

historiquement nulles et les exportations sont assez modestes (191 millions d'euros en 2015) tant par rapport aux principaux exportateurs mondiaux (Mexique et Australie) que comparativement aux exportations de viande bovine ; ces dernières étant vingt-cinq fois plus élevées en valeur. Ces exportations de bovins vivants sont destinées principalement au Venezuela (63\% du total), au Liban $(20 \%)$ et à la Jordanie $(10 \%)$.

Le Brésil n'importe presque pas de viande bovine, seulement quelques milliers de tonnes depuis l'Argentine et l'Uruguay. Les exportations sont passées, quant à elles, de 416300 tec en 2000 (pour un montant de 855 millions d'euros) à 1,69 million de tonnes en 2015 (pour un montant de 5,1 milliards d'euros). Le niveau record des exportations en volume a été atteint dès 2006 avec 1,87 million de tec (figure 3 ). À cette date, le Brésil occupait le premier rang mondial des pays exportateurs. En 2015, les exportations brésiliennes se font à $82 \%$ (en volume) sous la forme de viandes bovines congelées, $10 \%$ de viandes fraîches désossées et $8 \%$ de viandes transformées (préparations). Au fil des quinze dernières années, le panel de clients du Brésil en viande bovine s'est diversifié, avec notamment une baisse du poids relatif de l'UE et une montée en puissance des pays asiatiques. En se concentrant et en diversifiant leurs clientèles, les opérateurs brésiliens disposent désormais de débouchés complémentaires pour toutes les pièces d'une même carcasse, ce qui permet d'améliorer l'efficacité économique globale de la filière.
Les exportations à destination de HongKong et de la Chine ont considérablement progressé, surtout depuis 2011. Elles sont passées (en cumulant ces deux destinations) de 38700 tec en 2000 à 440500 tec en 2015, année record. Cette zone géographique représente désormais le quart des exportations brésiliennes de viande bovine tant en volume qu'en valeur. Avec 315300 tec importées en 2015, HongKong occupe le premier rang des importateurs en provenance du Brésil devant l'Égypte (245000 tec en 2015) et la Russie. Les importations russes, qui varient fortement d'une année à l'autre, ont atteint leur point culminant en 2007 (585 700 tec), pour retomber à 222700 tec en 2015. Avec 139100 tec, l'UE arrive en quatrième position des clients. Les importations européennes ont fortement baissé depuis leur pic de 2006 (521 700 tec), sous l'effet de la hausse du prix de vente de la viande bovine brésilienne (prix tiré par une consommation intérieure soutenue), de la baisse de la consommation dans l'UE et, surtout, de la modification des règles sanitaires imposées au Brésil à compter du 31 janvier 2008. Dans la continuité d'une visite d'inspection vétérinaire menée en 2007 et considérant que les normes de traçabilité étaient insuffisantes alors que ce pays est partiellement affecté par la maladie de la fièvre aphteuse, l'UE a limité de façon drastique le nombre des exploitations brésiliennes habilitées à exporter vers le marché européen. Selon l'OIE, quelques États brésiliens (dont Santa Catarina) qui ne pratiquent pas la vaccination sont cependant reconnus comme zone indemne de fièvre aphteuse. Les 
autres pays clients du Brésil sont, en 2015 , l'Iran (127 100 tec), la Chine (125 200 tec), le Venezuela (122 000 tec), le Chili (84 300 tec) et les États-Unis (39 200 tec).

\section{4 / Les États-Unis}

Avec une population de 325 millions d'habitants en 2016 (4,3\% de la population mondiale) et une surface agricole de 408 millions d'hectares (dont 38\% de terres arables), les États-Unis sont le premier pays producteur agricole au monde. Ils occupent le premier rang pour plusieurs productions agricoles dont le maïs grain, le soja, la viande de volaille et la viande bovine. Avec plus du double de terres agricoles que l'UE, mais grâce à une forte mécanisation, les États-Unis ont cinq fois moins d'exploitations agricoles (soit environ deux millions). Au travers du « Farm Bill », l'agriculture américaine bénéficie d'une politique agricole plus structurée que dans d'autres pays concurrents (dont l'Australie) et de systèmes assurantiels permettant aux exploitations de faire face, du moins pour une part, aux effets négatifs de la volatilité accrue des prix agricoles (Bureau 2013). Essentiellement de type familial (90\%), les exploitations comptent pour $2 \%$ des actifs. Si l'agriculture ne contribue que pour $1,3 \%$ au produit intérieur brut, le complexe agroalimentaire revêt une importance commerciale stratégique. Alors que la balance commerciale globale des Etats-Unis est très déficitaire ( -475 milliards d'euros en 2015), le solde du secteur agroalimentaire est positif (18 milliards d'euros à la même date). Si les échanges des ÉtatsUnis avec les deux autres membres de l'ALENA (accord de libre-échange nordaméricain entré en application à compter du 1 er janvier 1994), à savoir le Canada et le Mexique, sont historiquement élevés (un tiers de leurs importations agroalimentaires), les pays asiatiques occupent une place croissante dans les exportations. Au regard de déclarations récentes, l'élection de Donald Trump pourrait cependant entraîner certaines modifications dans les stratégies commerciales des USA. Ainsi, par exemple, le Président a ouvertement critiqué l'ALENA en citant le cas des produits laitiers canadiens. De même, un décret signé le 23 janvier 2017 stipule que les Etats-Unis entendent se désengager du traité multilatéral de libre-échange envisagé avec les pays asiatiques (le « Trans-Pacific Partnership Agreement »).

Concernant plus spécifiquement le secteur bovin, les États-Unis occupent une place majeure à l'échelle mondiale en étant, d'une part, le premier pays producteur $(16 \%$ de la production mondiale), consommateur et importateur de viande bovine et, d'autre part, le quatrième pays exportateur (Institut de
l'Élevage 2015a). Avec un cheptel de 89 millions de têtes, les États-Unis produisent 10,8 millions de tec de viande bovine en 2015 . Le secteur bovin-viande, qui compte 620000 exploitations spécialisées, est assez concentré dans la partie centrale du pays ; les cinq États les plus représentés sont le Texas (13,1\% du cheptel national en 2015), le Nebraska (7\%), le Kansas $(6,7 \%)$, la Californie $(5,7 \%)$ et l'Oklahoma $(5,1 \%)$.

La production de viande bovine n'est cependant pas en hausse puisqu'elle atteignait déjà 12,1 millions de tec en 2000 (Institut de l'Élevage 2015a). En 2004, l'apparition de l'épizootie ESB avait déjà affecté les abattages du fait notamment de l'arrêt des importations de bovins vivants en provenance du Canada. Après un retour à un niveau de croisière (environ 12 millions de tec) entre 2006 et 2010 , les abattages ont de nouveau reculé jusqu'en 2015 sous l'influence d'une baisse du cheptel allaitant (décapitalisation imputable à la sécheresse dans différents États et à l'augmentation du prix des matières premières), du plafonnement des poids de carcasse et d'une diminution des importations de bovins vivants en provenance du Mexique. Le cheptel de vaches allaitantes a atteint son niveau le plus bas des quinze dernières années en 2014 pour remonter à un peu plus de 30 millions de têtes en 2016. La croissance de la production laitière américaine s'accompagnant d'une amélioration de la performance zootechnique, le cheptel de vaches laitières $(9,1$ millions de têtes en 2015) est, quant à lui, relativement stable depuis 2000 (USDA 2017a). La contribution du secteur laitier à l'offre nationale de viande bovine reste donc globalement modeste, du moins comparativement à la situation européenne.

Au niveau des systèmes productifs, la production de viande bovine américaine fait l'objet d'une segmentation forte entre les activités de naissage et celle d'engraissement (USDA 2011). Du fait de la faible rentabilité à l'hectare des activités de naissage, basées sur un mode de production très extensif, les exploitations impliquées sont logiquement situées dans des zones où les terres labourables permettant le développement des cultures de vente sont rares. De leur naissance jusqu'au sevrage, les bovins sont élevés au pâturage dans des conditions peu coûteuses tant au niveau des charges opérationnelles (aliments, frais vétérinaires...) que des infrastructures dédiées (bâtiments). Les élevages naisseurs sont cependant très sensibles aux disponibilités fourragères, lesquelles tiennent pour une grande part au facteur climatique. Ils sont également économiquement soumis aux rapports de prix qui s'établissent tout au long de la filière, le prix de vente des broutards étant influencé par la demande des acteurs de l'engraissement dont la rentabilité est pour partie conditionnée à l'évolution du prix d'achat des aliments. Avant de basculer dans une phase d'engraissement, les broutards bénéficient d'une étape intermédiaire de plusieurs mois, dite " la repousse ", réalisée parfois dans des exploitations spécialisées pour cette activité. La phase d'engraissement recourt à un modèle de production radicalement différent, car très intensif (forte concentration de bovins à l'hectare) et basé sur l'utilisation d'aliments issus de cultures (maïs ensilage, maïs grain, soja...) ou de l'industrie agroalimentaire (dont les coproduits de la filière éthanol). Les élevages d'engraissement ou feed lot de grande taille jouent un rôle décisif dans la production américaine (Galyean et al 2011). En effet, en 2015, et d'après l'USDA, les 2190 feed lot de plus de 1000 têtes concentrent 16,9 millions de bovins. Si ces élevages intensifs sont fortement dépendants du prix de l'alimentation, ils sont peu coûteux à la tonne de viande bovine produite pour les bâtiments, ce qui constitue un avantage des États-Unis sur les marchés extérieurs.

Au niveau commercial, les États-Unis sont, de loin, le premier importateur mondial de bovins vivants au monde $(1,97$ millions de têtes, hors cheptel reproducteur, en 2015). Ce pays est nettement déficitaire en valeur (- 1,93 milliard d'euros en 2015), son niveau d'importations (2,03 milliards d'euros) étant vingt fois supérieur à celui de ses exportations $(95$ millions d'euros en 2015). Le déficit observé en 2015, qui s'explique en partie par la décapitalisation du cheptel allaitant, est le plus important des quinze dernières années. En raison de la fermeture momentanée des échanges avec le Canada suite à la découverte d'un cas d'ESB sur une vache laitière importée du Canada, le déficit a été plus réduit en 2004 (- 435 millions d'euros de solde). Plus de $80 \%$ des exportations étatsuniennes de bovins vivants sont destinées aux quatre pays suivants : le Mexique, le Canada, la Russie et la Turquie. Les importations proviennent, quant à elles, exclusivement du Canada $(63 \%$ des approvisionnements en valeur en moyenne sur la période 2000 à 2015) et du Mexique (37\%). Si le Mexique exporte surtout des bovins maigres, les deux tiers des bovins exportés par le Canada sont destinés à être abattus aux ÉtatsUnis.

Ces courants d'échanges entre les membres de l'ALENA ne doivent pas masquer l'existence de certaines tensions. Ainsi, par exemple, le Canada et le Mexique ont engagé fin 2008 une procédure auprès de l'organe de règlement des différends à l'OMC relativement à la loi américaine dite « Country 
of Origin Labelling ». Celle-ci permet l'introduction d'un étiquetage sur les viandes de bœuf et de porc en fonction de leurs origines (Greene 2015). D'après ces deux pays frontaliers, cette loi entraînait un lourd préjudice pour leurs exportations dans la mesure où les consommateurs américains donnaient, dans ce cadre, leur préférence aux bovins nés, engraissés et abattus aux États-Unis. Après plusieurs années de procédures juridiques, l'OMC a donné raison en décembre 2015 au Canada, pays qui a été en mesure d'imposer des surtaxes sur les exportations américaines pour un montant annuel de 1,05 milliard de dollars. Finalement, et à la satisfaction des négociateurs canadiens, cette loi américaine a été abrogée peu de temps plus tard pour permettre aux États-Unis d'échapper à ces sanctions. D'après certaines affirmations, la nouvelle administration américaine serait sensible à cette question et pourrait potentiellement réagir dans les mois/années à venir.

En dépit de sa situation de premier producteur mondial, les États-Unis assument un solde commercial négatif en viande bovine au titre de l'année 2015, tant en volume (-388000 tec) qu'en valeur (- 983 millions d'euros). Si la consommation intérieure globale de viande bovine, au demeurant négativement impactée par la hausse des prix de vente au consommateur, s'inscrit à la baisse (-12\% entre 2006 et 2015$)$, le niveau individuel reste parmi les plus élevés au monde (35 kg en équivalent carcasse par habitant et par an en 2015). En moyenne annuelle sur la période 2000 à 2015 , le solde en viande bovine a été de - 227000 tec. En raison de la baisse drastique de production (ESB), il avait chuté à son plus bas niveau en 2004 (- 1,3 million de tec); son niveau le plus élevé ayant été obtenu en 2013 (+359000 tec).

Les importations étatsuniennes de viande bovine, qui s'élèvent à 1,5 million de tec en 2015 pour un montant de 6,23 milliards d'euros, se font principalement sous la forme de viandes congelées $(59 \%$ du tonnage) et de viandes fraîches désossées (32\% du tonnage). Elles proviennent surtout des quatre pays suivants : l'Australie (38\% des volumes en 2015), la Nouvelle-Zélande (19\%), le Canada (19\%) et le Mexique (12\%). Les autres pays fournisseurs sont l'Uruguay $(4 \%)$, le Nicaragua (3\%), le Brésil $(2 \%)$ et le Costa Rica (1\%). Les importations en provenance de l'UE sont pratiquement inexistantes. Au cours de la période étudiée, les importations étatsuniennes de viande bovine ont varié de façon importante en fonction des évènements sanitaires (le maximum ayant été atteint en 2004 : 1,6 million de tec) et des disponibilités intérieures. Compte tenu des accords préférentiels passés entre les
États-Unis et l'Australie (AUSFTA), les importations de viande bovine seront facilitées à compter de 2022, en raison, d'une part, de l'augmentation programmée du contingent d'importations à tarif réduit (qui sera porté à 448210 tonnes contre 418000 tonnes en 2015) et, d'autre part, de l'annulation des tarifs intracontingentaires (de 26 à $0 \%$ ).

Bien que déficitaire en viande bovine et premier importateur mondial, les ÉtatsUnis se hissent au quatrième rang des exportateurs. En 2015, les exportations se sont élevées à 1,11 million de tec pour un montant de 5,25 milliards d'euros. Ainsi, le prix de vente à l'export de la viande bovine est estimé à 4,7 euros par $\mathrm{kg}$ en équivalent carcasse, soit un niveau supérieur au prix d'achat de la viande importée (4,1 euros par $\mathrm{kg}$ ). Les exportations sont destinées surtout au Japon (22\% des tonnages), au Mexique $(17 \%)$, au Canada (13\%) et à la Corée du Sud (12\%). Les autres clients sont l'Égypte (5\%), Taïwan (4\%), les Antilles et Bahamas (3\%) et l'UE (2\%). En raison de l'ESB et de la fermeture de leurs débouchés en Asie, les exportations avaient atteint un niveau minimal en 2004 (300 000 tec, dont les trois quarts étaient destinées au Mexique et Canada). Le point culminant a été atteint en 2000 (1,39 million de tec). Si le Japon est redevenu le premier client des États-Unis, les achats de ce pays ont considérablement varié, en fonction de la situation sanitaire. Ce pays, qui importait 533700 tec de viande bovine aux États-Unis en 2000, a totalement arrêté ses achats en
2004 et 2005 (figure 4) ; après avoir été remplacés par l'Australie, les États-Unis sont parvenus à revenir sur le marché japonais, mais pour des volumes nettement inférieurs (246000 tec en 2015) à ceux constatés avant la crise de l'ESB (la situation est semblable en Corée du Sud). Les exportations ont également baissé à destination du Mexique et, dans une moindre mesure, du Canada. Elles ont, en revanche, progressé vers HongKong (porte d'entrée vers la Chine continentale), Taïwan et l'Egypte.

Si les exportations de viande bovine étatsuniennes vers l'UE sont, et depuis fort longtemps, placées au centre de nombreux débats, elles restent marginales. Elles représentent, en effet, $1,7 \%$ des exportations étatsuniennes en moyenne annuelle sur la période 2000 à 2015 (soit 17000 tec). À compter de 1988, et au nom du principe de précaution, l'UE interdit l'arrivée sur son territoire du bœuf américain, considérant que le traitement aux hormones de croissance des bovins est susceptible de nuire à la santé de ses consommateurs. Dès 1996, cette décision a conduit les américains et les canadiens à porter plainte auprès de l'OMC qui, après la réalisation de plusieurs études scientifiques non concluantes sur les risques encourus, leur a donné raison. Ainsi, à partir de 1999, les ÉtatsUnis et le Canada ont eu l'opportunité d'appliquer des sanctions douanières sur de nombreux produits agroalimentaires européens importés (dont la viande bovine, la viande porcine, le Roquefort, le chocolat...), pour un montant annuel

Figure 4. Les exportations des États-Unis en viande bovine (Milliers de tec). (Source : BACl d'après Comtrade/Traitement INRA, SMART-LERECO).

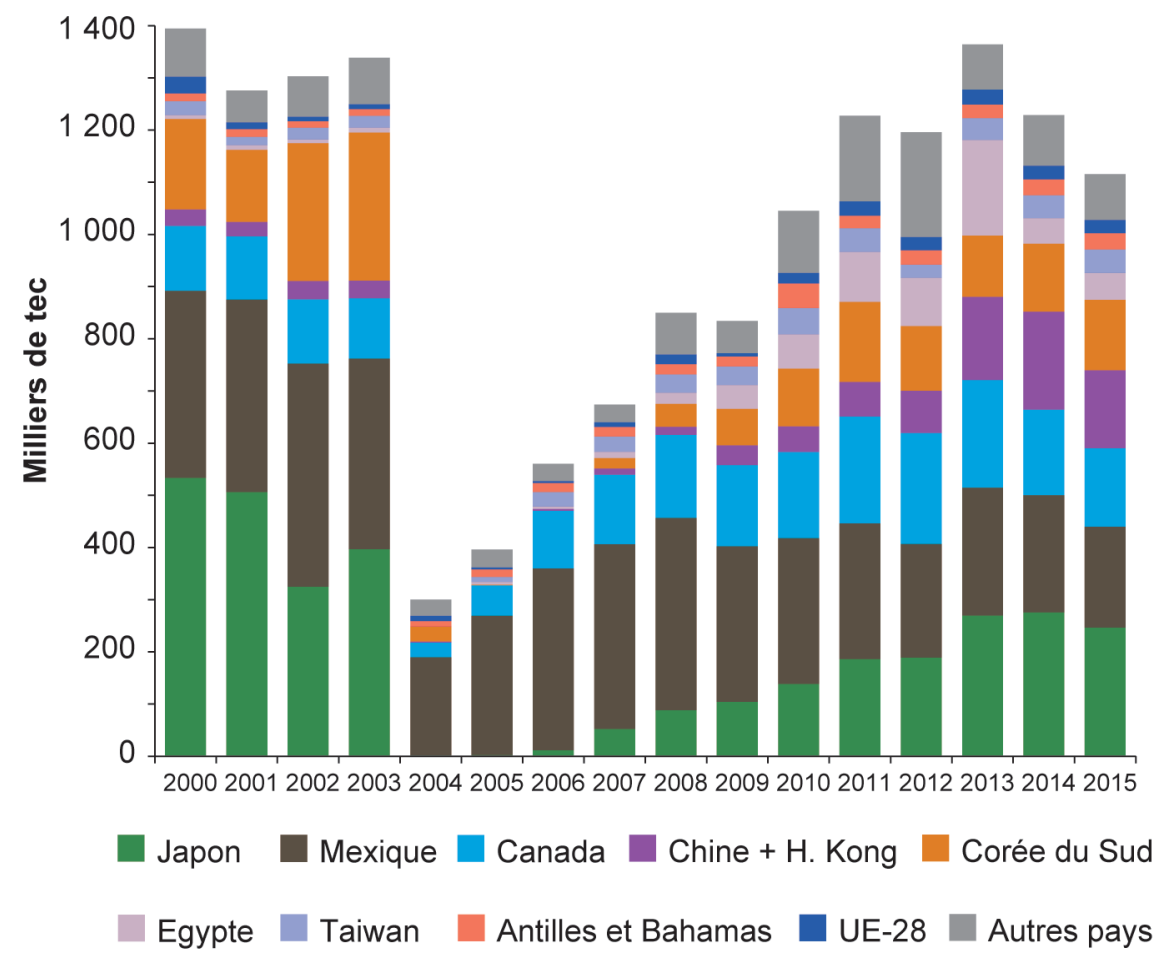


de, respectivement, 116,8 millions de dollars étatsuniens et 11,3 millions de dollars canadiens. À compter de mai 2009 , un nouvel accord a été négocié entre l'UE et les gouvernements étatsunien et canadien. Celui-ci comportait trois engagements : le maintien de l'interdiction d'importation par l'UE de viandes bovines aux hormones en provenance des États-Unis ; le renoncement des ÉtatsUnis et du Canada à l'application de sanctions financières sur les importations européennes ; l'attribution par l'UE d'un contingent à l'importation (20 000 tonnes pendant les trois premières années, puis 45000 tonnes à partir de la quatrième année) pour le bœuf américain de haute qualité (Hilton beef), non sujet à l'administration d'hormones de croissance. Cette viande de haute qualité est achetée désossée et réfrigérée et elle est généralement commercialisée dans une restauration de luxe. En 2017, et avant même l'arrivée en fonction du nouveau Président, il apparaît que l'Administration des États-Unis conteste les conséquences de cet accord. Le contingent tarifaire dit « panel aux hormones " s'est en effet ouvert à d'autre pays fournisseurs (Uruguay, Argentine, Australie...) en vertu du principe de la nation la plus favorisée. Par ailleurs, les canadiens pourraient bénéficier d'une consolidation de leur contingent avec l'UE dans le cadre de l'accord bilatéral du CETA («Comprehensive Economic and Trade Agreement»).

\section{2 / Les principaux impor- tateurs mondiaux dans le secteur bovin}

Cette deuxième partie s'intéresse à la situation spécifique des trois pays ou regroupements de pays les plus importateurs de viande bovine, à savoir : i) le regroupement de la Chine, de Hong-Kong et du Vietnam dont les importations globales s'élèvent 1,76 million de tec en 2015 (soit plus que les États-Unis) et sont en forte croissance. Ces trois pays sont traités ici comme une seule entité géographique dans la mesure où il est difficile d'entrevoir, du moins au travers des statistiques douanières disponibles, la part des importations du Vietnam et de Hong-Kong qui est ensuite commercialisée en Chine ; ii) le cas du Japon, dont les importations ont plutôt baissé depuis 2000 (704 000 tec en 2015); iii) le cas de la Russie (615 000 tec en 2015) où l'application récente d'un embargo modifie les courants d'échanges. Les autres principaux pays importateurs, dont l'Égypte, la Corée du Sud, l'UE-28, le Canada, le Mexique, l'Arabie Saoudite et le Venezuela, ne feront pas l'objet de points spécifiques.

Cette partie porte sur les grands importateurs de viande bovine et non pas ceux de bovins vivants. Les importations de bovins vivants sont, quant à elles, dominées par les États-Unis (1,97 million de têtes en 2015) qui devancent de très loin l'Indonésie (520 000 têtes), la Turquie (360 000 têtes), le Vietnam (320 000 têtes), le Liban (300 000 têtes), l'Égypte (160000 têtes) et l'Israël (160000 têtes).

\section{1 / La Chine, étendue à Hong- Kong et au Vietnam}

Avec ses 1,38 milliard d'habitants (en incluant Hong-Kong ${ }^{1}$; le Vietnam compte 94 millions d'habitants), la Chine est le premier marché mondial de la consommation alimentaire. Avec 9\% des surfaces labourables au monde et $7 \%$ des ressources en eau, la Chine doit assurer l'alimentation de $21 \%$ de la population mondiale ce qui constitue un challenge conséquent pour son agriculture qui représente environ $10 \%$ du produit intérieur brut. Grâce à une politique de soutien des prix intérieurs, au développement des techniques et à l'essor de la mécanisation, la Chine parvient à dynamiser ses productions végétales, à tel point que ce pays est aujourd'hui le premier producteur mondial de blé, de riz, de pommes de terre et le deuxième en maiis. Les productions animales (y compris l'aquaculture), qui représentent un tiers de la valeur ajoutée agricole du pays, se sont elles aussi développées, plus cependant en viande porcine (la moitié de la production mondiale) et en viande de volaille (17\%) que dans le secteur laitier $(5 \%)$. Ce développement des filières animales, qui a été permis grâce aux achats de soja en provenance du continent américain (Jamet et Chaumet 2016), se révèle cependant insuffisant pour couvrir l'intégralité des besoins intérieurs (Chaumet et Pouch 2017). Bien que la Chine occupe la troisième place des zones exportatrices de biens agroalimentaires derrière l'UE et les États-Unis, sa balance commerciale demeure largement négative (- 52 milliards d'euros en 2015). Outre son accession à l'OMC à compter de décembre 2001, la Chine a signé de nombreux accords commerciaux bilatéraux qui contribuent à une ouverture accentuée de son marché.
Le cheptel de bovin de la Chine s'élève, en 2015 , à 100 millions de têtes, dont 15,5 millions de vaches laitières et 50,7 millions de vaches allaitantes (USDA 2017b). D'après les données de l'OCDE, la production chinoise de viande bovine, qui était traditionnellement faible, a progressé en passant de 1,2 million de tec en 1990, à 5,3 millions de tec en 2000 et 7 millions de tec en 2015 , soit $8 \%$ du tonnage total des viandes produites localement. Au Vietnam, la production bovine est estimée à 500000 tec pour un cheptel de 8 millions de têtes. Le développement de la production bovine chinoise rencontre de sérieuses difficultés (Guanghong et al 2012, Han et al 2016 ) : une forte dispersion du cheptel dans un très grand nombre d'exploitations de très petite taille ; une baisse du cheptel de vaches reproductrices; une mauvaise valorisation en viande bovine du potentiel des animaux de réforme issus du secteur laitier ; un faible niveau de structuration de la filière, même si certaines entreprises ont tenté de développer des ateliers de grande taille ; un dispositif perfectible dans le domaine de la génétique animale ; un manque de technicité dans de nombreux élevages, ce d'autant qu'une part des bovins est encore utilisée comme animaux de traction ; un faible potentiel de rendement des surfaces fourragères, notamment dans les zones pastorales où la gestion du pâturage n'est pas toujours optimale, etc. (Huang et al 2015).

Ainsi, la production chinoise et vietnamienne de viande bovine ne parvient pas à satisfaire la demande locale. Plus que dans d'autres pays en développement (dont l'Inde), la consommation annuelle de viande par habitant a considérablement augmenté en Chine (Zhou et al 2012). Limitée seulement à $3 \mathrm{~kg}$ au début des années soixante, dans une période où elle était contrôlée par les pouvoirs publics, elle atteint aujourd'hui près de $60 \mathrm{~kg}$, dont environ $6 \mathrm{~kg}$ de viande bovine. Si les chinois consomment d'abord de la viande porcine (environ $40 \mathrm{~kg}$ ), ils diversifient progressivement la structure de leur régime alimentaire.

Dans un tel cadre, les importations de la Chine (avec Hong-Kong et le Vietnam) tiennent une place désormais importante dans le commerce international. Elles sont passées de 242000 tec en 2000 à 1,76 million de tec en 2015 (figure 5). Ainsi, la progression des importations de cette zone est exceptionnelle et contribue pour une grande part à la dynamisation des

\footnotetext{
${ }^{1}$ Colonie britannique à partir du traité de Nankin (1842), Hong-Kong a été rétrocédée à la Chine en 1997. Selon les termes de la déclaration sino-britannique commune, la Chine a promis que Hong-Kong garderait une relative autonomie jusqu'à au moins 2047. Cette clause permet à cette région d'adopter des droits de douane attractifs pour les exportateurs de viande bovine et de mettre en œuvre une réglementation sanitaire distincte de celle de la Chine continentale. Le port de Hong-Kong étant classé au cinquième rang mondial pour son trafic, une part importante des importations de cette zone (7 millions d'habitants) est destinée, in fine, au reste de la Chine.
} 
Figure 5. Les principaux pays importateurs de viande bovine (Milliers de tec). (Source : BACI d'après Comtrade/Traitement INRA, SMART-LERECO).

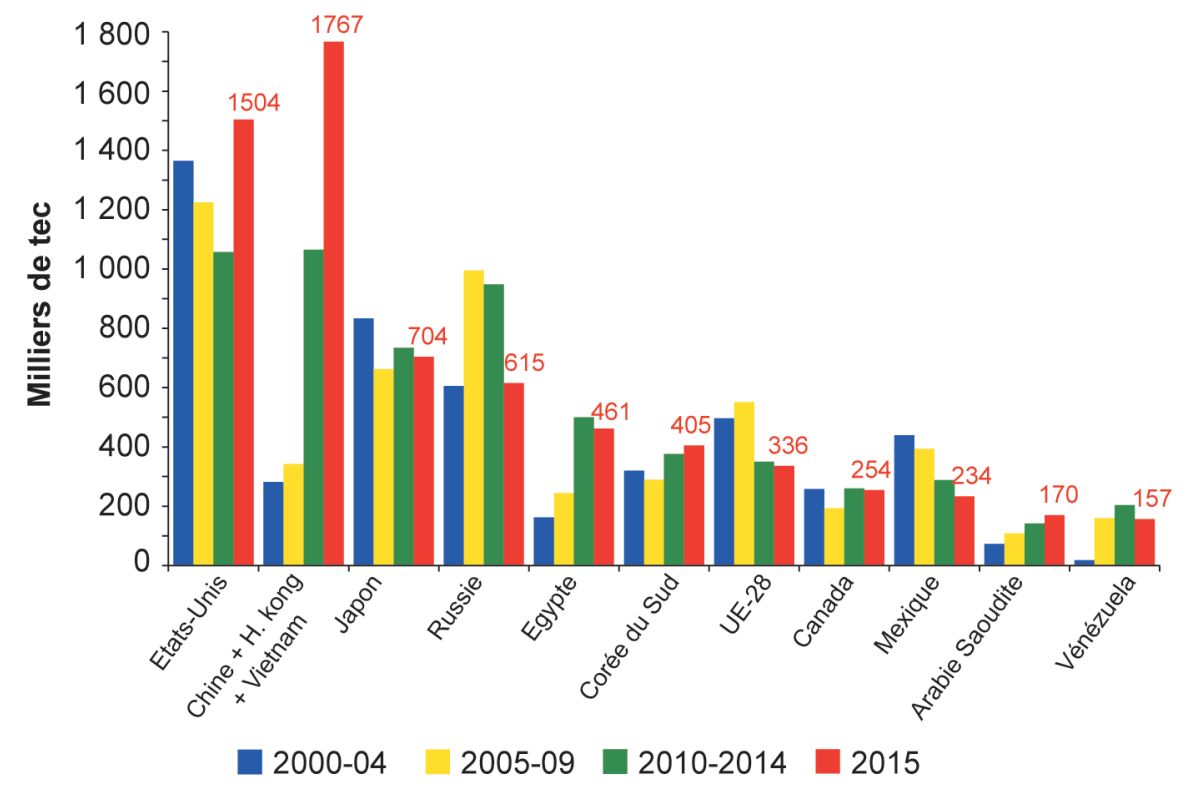

flux internationaux de viande bovine. Le volume annuel des importations, qui n'a jamais excédé les 700000 tec entre 2000 et 2011 , a considérablement augmenté au cours des cinq dernières années pour atteindre son maximum en 2015. Elles proviennent à $95 \%$ de viande congelée, $4 \%$ de viande transformée et seulement $1 \%$ de viande réfrigérée.

En 2015, l'Inde est le principal fournisseur de cette zone. Pour contourner les réglementations chinoises en vigueur, la viande bovine indienne est exportée vers le Vietnam pour être ensuite commercialisée en Chine via des canaux illégaux. En effet, ce pays ne bénéficie pas des licences imposées par les services centraux vétérinaires et obligatoires pour espérer vendre de la viande bovine aux chinois. Les canaux de valorisation de la viande indienne sur le marché chinois sont cependant souvent différents de leurs compétiteurs. Jusqu'à une période récente (2015), sept pays seulement avaient la possibilité d'exporter directement de la viande bovine vers la Chine (Liu et Iqbal 2016) : l'Australie, la Nouvelle Zélande, le Brésil, l'Uruguay, le Canada, l'Argentine et le Costa Rica. De fait, et outre l'Inde, les principaux exportateurs de viande bovine vers la Chine sont les pays de l'Océanie et du Mercosur. Récemment, la réglementation chinoise vient cependant de s'assouplir. Les États-Unis, qui exportaient de la viande bovine uniquement vers Hong-Kong (127000 tec en 2015), mais pas vers la Chine continentale, ont en effet bénéficié en septembre 2016 d'une levée partielle des restrictions chinoises à l'importation. En février 2017, le Gouvernement chinois a également annoncé la levée partielle de l'embargo qui frappait la viande bovine française à la suite de la crise de
l'ESB (maladie dite de la vache folle). Cette décision a été prise près de neuf ans après la reconnaissance par l'Organisation mondiale de la santé animale (OIE) du statut de pays « à risque maîtrisé » de la France. Le chemin paraît encore long pour les acteurs français (au moins deux ans pour l'agrément des effectivement démarrer.

\section{2 / Le Japon}

Avec une population de 127 millions d'habitants en 2015 pour 4,5 millions d'hectares de surface agricole, le Japon connait des difficultés structurelles d'approvisionnement alimentaire, ce en dépit des politiques publiques volontaristes adoptées (OCDE 2009) : les surfaces arables sont limitées ; l'urbanisation et la forêt (les deux tiers du territoire) entrent en concurrence avec le foncier agricole ; les parcellaires sont très morcelés ; les exploitations sont de petite taille ; la population agricole est vieillissante (les trois quarts des agriculteurs ont plus de 60 ans) ; la production agricole a reculé de l'ordre de $30 \%$ depuis 1995. Ainsi, l'agriculture, qui mobilise $4 \%$ de la population active et contribue pour $1,2 \%$ au produit national brut, ne parvient à satisfaire qu'environ $40 \%$ des besoins caloriques du pays (Poncet et Hofferer 2016). Le Japon est ainsi le quatrième importateur mondial de biens agroalimentaires. Ses importations (49 milliards d'euros en 2015) dépassant de loin ses exportations (7 milliards d'euros), le pays du soleil-levant est très déficitaire (- 42 milliards d'euros). Les trois principaux fournisseurs sont les États-Unis (25\% des importations), l'UE (14\%) et la Chine (12\%). Les viandes et les prooutils...) avant que les flux ne puissent duits carnés représentent $21 \%$ des importations agroalimentaires du pays.

Le secteur bovin japonais compte un cheptel de 3,8 millions de têtes, dont 750000 vaches laitières et 580000 vaches allaitantes (USDA 2016). Les bovins Wagyu (y compris les réputés bœufs de Kobé), dont la qualité de la viande est souvent mise en avant (Gotoh et al 2014), compte pour environ la moitié des abattages qui s'établissent à environ 1,1 million de têtes en 2015. La production intérieure de viande bovine s'élève à 481000 tec en 2015 , soit un niveau nettement inférieur à celui de la consommation intérieure (1,2 million de tec), laquelle s'inscrit en légère baisse depuis quelques années. Le prix attractif des autres protéines animales, la hausse des prix de la viande bovine et la légère baisse de la population nipponne depuis 2010 (moins un million d'habitants) confortent cette tendance. Au Japon, la consommation individuelle de viande bovine ( $9 \mathrm{~kg}$ par habitant et par an) est proche de la moyenne mondiale et elle est inférieure à celle de viande porcine $(20 \mathrm{~kg})$ ou de viande de volaille $(21 \mathrm{~kg})$.

Les exportations du Japon dans le secteur bovin sont marginales. Elles se sont élevées, en moyenne annuelle sur la période 2000 à 2015 , à seulement 25 millions d'euros, dont $30 \%$ à destination du Cambodge, 28\% de HongKong, $17 \%$ des États-Unis et $4 \%$ de l'UE. Si le Japon n'importe pratiquement pas de bovins vivants, il se positionne au quatrième rang mondial des importateurs de viande bovine. Les importations japonaises se sont élevées, en moyenne annuelle sur la période 2000 à 2015, à 740900 tec pour un montant de 2,12 milliards d'euros. Au cours de la période étudiée, les importations ont atteint leur pic en 2000, avec 1,04 million de tec. Suite à la fermeture du marché américain pour des raisons sanitaires (FranceAgriMer 2012; voir supra), les importations ont chuté à 610000 tec en 2004. Elles ont peu évolué depuis lors, atteignant 704000 tec en 2015. Ces dernières relèvent à $55 \%$ de viandes congelées, $38 \%$ de viandes réfrigérées désossées, $5 \%$ d'abats et $2 \%$ de viandes transformées. Le prix des produits importés a augmenté au cours des dernières années en passant de 267 euros/tec en 2010 à 407 euros/tec en 2015. Les États-Unis qui représentaient $51 \%$ des approvisionnements japonais en 2000 en assurent désormais $35 \%$ (figure 6) ; ils ont ainsi été relégués en seconde position derrière l'Australie dont les parts de marché sont passées, dans le même temps, de $42 \%$ à $56 \%$. L'Australie a bénéficié, d'une part, d'une évolution plus favorable de sa parité monétaire vis-à-vis du dollar étatsunien et, d'autre part, d'une baisse des tarifs douaniers dans le cadre de ses accords 
Figure 6. Les importations du Japon en viande bovine (Milliers de tec) (Source : BACI d'après Comtrade/Traitement INRA, SMART-LERECO).

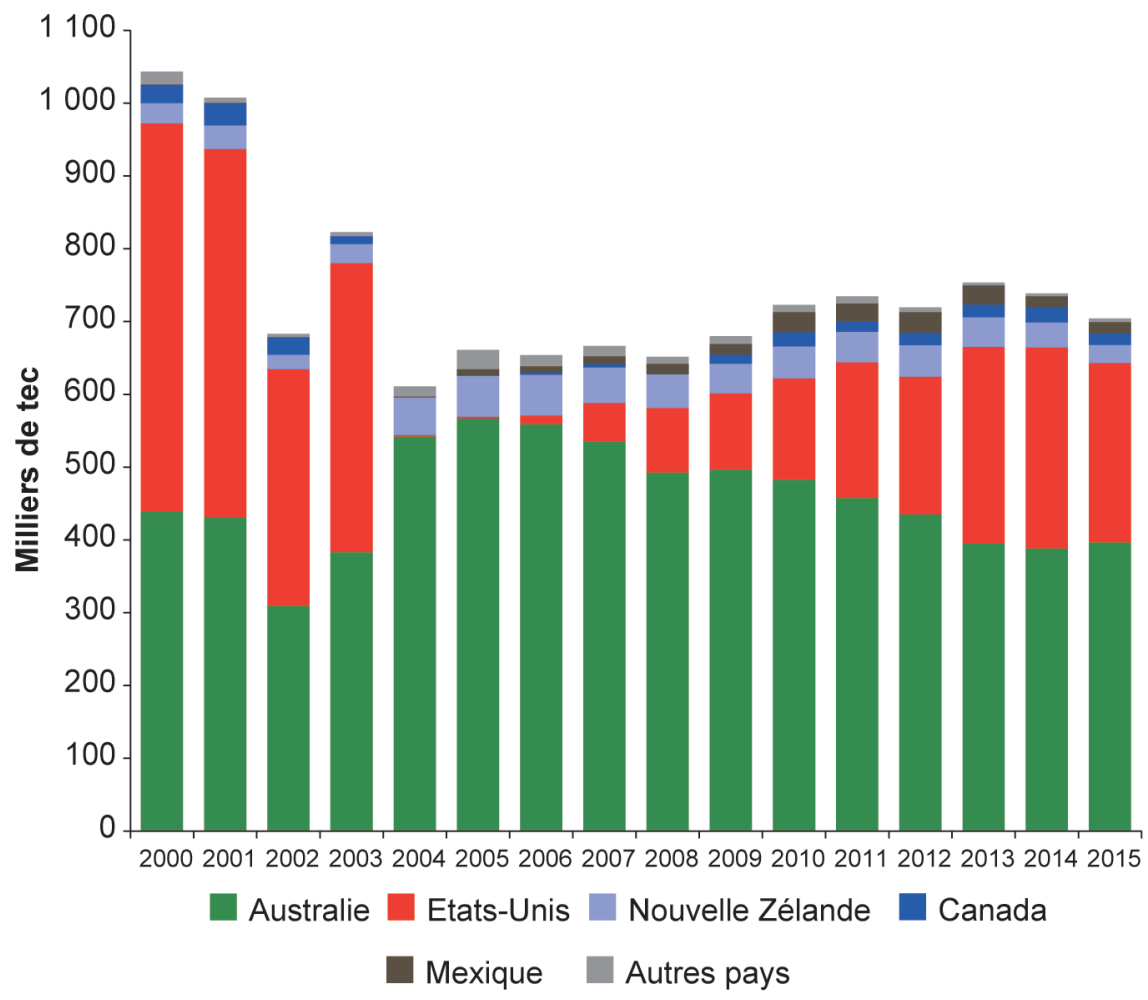

bilatéraux avec le Japon. Les autres pays fournisseurs du Japon sont la NouvelleZélande $(3,5 \%$ en 2015$)$, le Canada $(2,3 \%)$ et le Mexique $(2,2 \%)$.

\section{3 / La Russie}

Avec une population de 146 millions d'habitants en 2016, la Russie bénéficie d'une surface agricole utile conséquente (216 millions d'hectares), mais néanmoins modeste au regard de la surface totale du pays $\left(17,1\right.$ millions de $\left.\mathrm{km}^{2}\right)$. En dépit de l'existence de terres noires fertiles au sud, l'agriculture doit s'adapter à un climat continental rigoureux laissant place à une période végétative restreinte et à des sécheresses fréquentes (Cordonnier et Hervé, 2003). La place de l'agriculture dans l'économie nationale a considérablement baissé au cours des dernières décennies pour ne représenter que $4 \%$ du produit intérieur brut en 2015. Après la dissolution de l'Union soviétique en 1991, les grandes fermes collectives et étatiques ont dû faire face à la perte soudaine de canaux de commercialisation et d'approvisionnement garantis par l'État et à un environnement juridique changeant qui s'est soldé par une restructuration intense du secteur et une baisse de la production, notamment dans le secteur bovin. Après une période de stabilisation de l'offre agricole, celleci est désormais encouragée par les pouvoirs publics dans le cadre de son programme de développement agricole adopté pour la période 2013-2020. En 2015-2016, l'industrie a atteint ses objec- tifs d'autosuffisance en viande de porc et de volaille, mais pas en viande bovine et en produits laitiers où les investissements sont plus limités. Membre de l'OMC depuis 2012, la Russie est une grande puissance exportatrice en céréales. Si les importations agroalimentaires (23,8 milliards d'euros en 2015) s'inscrivent à la baisse depuis l'application en août 2014 d'un embargo sur les produits en provenance de plusieurs pays (UE, États-Unis, Canada, Australie, Norvège) en représailles aux mesures prises par ces pays suite à la guerre en Ukraine (annexion russe de la Crimée et implication dans les combats au Dombass), le solde agroalimentaire reste déficitaire (- 9 milliards d'euros). L'embargo a certes eu un impact négatif sur les exportations agroalimentaires de l'UE à destination de la Russie (McEldowney 2016), mais il n'a pas permis un développement de la production agricole intérieure aussi conséquent que ce qui pouvait être escompté par les autorités russes. La dépréciation du rouble et les conditions plus difficiles d'accès aux financements ont, en effet, fragilisé les investissements dans le secteur agricole, notamment dans le secteur bovin.

Le cheptel russe de bovins a considérablement baissé, passant de 59 millions de têtes en 1990, à 28 millions de têtes en 2000 et 18 millions de têtes en 2015 (Prikhodko et Davleyev 2014). Il est désormais composé de 7,8 millions de vaches laitières et 500000 vaches allaitantes. Les abattages de bovins, qui relè- vent surtout du cheptel laitier, représentent 1,34 million de tec en 2015 (USDA, 2017c). Après avoir baissé entre 1990 et 2000 , la consommation de viandes par habitant a progressé au cours de la période étudiée pour retrouver sa situation initiale, soit environ $65 \mathrm{~kg}$ par habitant et par an, dont $17 \mathrm{~kg}$ de viande bovine. Dans un contexte économique difficile pour les ménages russes, qui se caractérise par une dépréciation de la monnaie, une hausse des taux d'intérêt et l'inflation, cette hausse récente de la consommation concerne surtout les viandes de volaille et de porc.

Les exportations de la Russie dans le secteur bovin sont très faibles $(27$ millions d'euros en 2015) et destinées surtout au Kazakhstan, à la Biélorussie, à la Géorgie et à l'Ukraine. En raison de la baisse de la production, les importations dans le secteur bovin sont, en revanche, historiquement élevées. Les importations russes de bovins vivants, qui s'élèvent à 140000 têtes pour un montant de 151 millions d'euros en 2015 (dont 55\% de bovins reproducteurs), proviennent essentiellement de l'UE (46\%), de l'Australie $(40 \%)$ et des États-Unis (10\%). Elles ont augmenté au cours de la période récente, surtout au bénéfice de l'Australie; elles n'atteignaient, en effet, que 10 millions d'euros en moyenne annuelle au cours de la période 2000 à 2005 . Les importations de viande bovine, qui se sont élevées à 835000 tec en moyenne annuelle sur la période 2000-2015, ont fortement fluctué : elles sont passées de 392000 tec en 2000 à 1,12 million de tec en 2008 pour redescendre à 615000 tec en 2015.

La provenance des importations russes a évolué en fonction des disponibilités en viande bovine dans les pays exportateurs et des relations bilatérales existantes en matière sanitaire (figure 7). Ainsi, au début des années 2000, les approvisionnements se faisaient surtout en UE (41\% des volumes) et en Ukraine (39\%). Les trois principaux pays européens impliqués étaient l'Allemagne, l'Irlande et la Lituanie. En 2008, année record pour les importations russes, les principaux fournisseurs étaient le Brésil (44\%), puis loin derrière l'Uruguay $(10 \%)$, le Paraguay (9\%), l'Argentine (9\%), l'Australie (8\%) et l'UE (7\%). En 2015, l'application de l'embargo a profondément modifié les équilibres entre pays fournisseurs. Les importations proviennent désormais essentiellement du Brésil (36\%), de la Biélorussie (24\%), du Paraguay $(22 \%)$ et d'Argentine (4\%). L'UE, les Etats-Unis et l'Australie sont ainsi privés de ce débouché. Suite à la signature d'un décret en 2016 par le Président Vladimir Poutine, l'embargo russe devrait se poursuivre au moins jusqu'à la fin de l'année 2017. 
Figure 7. Les importations de la Russie en viande bovine (Milliers de tec). (Source : Comext/Traitement INRA, SMART-LERECO).

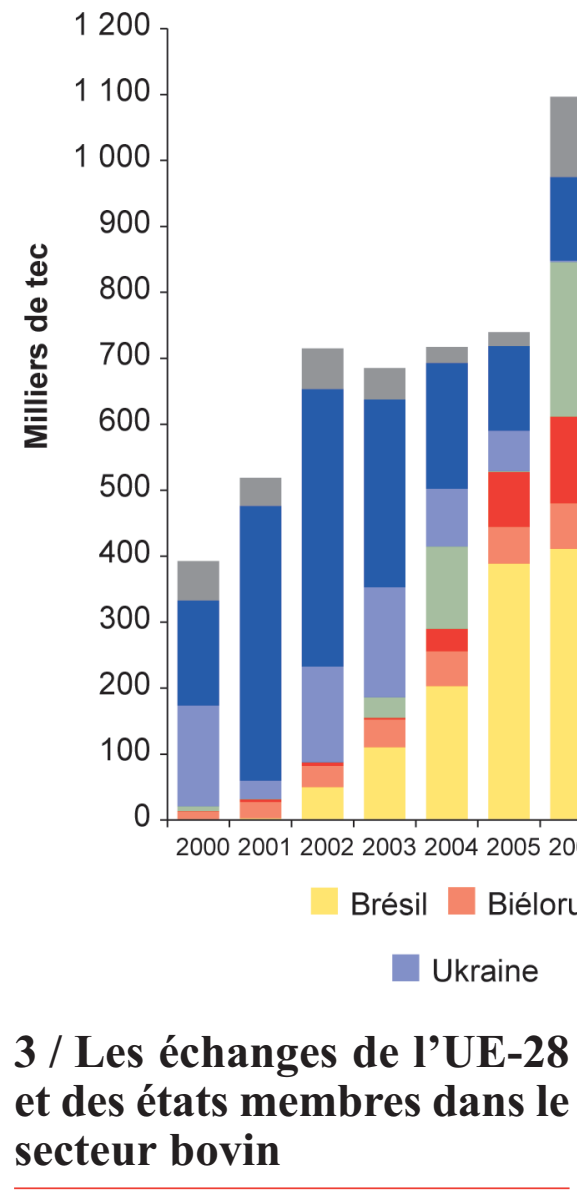

Avec une superficie agricole utile de 178 millions d'hectares (dont 60\% de terres arables), un climat favorable, un potentiel agronomique élevé et une Politique Agricole Commune (PAC) protectrice, l'UE est devenue une grande puissance agricole. La production agricole finale européenne, qui atteint 411 milliards d'euros en 2015 , résulte à $39 \%$ des productions animales et $8 \%$ du secteur bovin, hors activité laitière. L'UE est à la fois la première zone exportatrice (129 milliards d'euros en 2015) et importatrice (114 milliards d'euros en 2015) de produits agroalimentaires. L'UE est excédentaire, ou tout près de l'autosuffisance, pour de nombreux produits agricoles des zones tempérées, dont les céréales, le lait, la viande porcine, la viande bovine, etc. Les 506 millions d'européens se nourrissent surtout de produits alimentaires européens, moyennant l'existence de flux importants de marchandises entre États membres.

Le cheptel européen de bovins, qui s'élève à 89 millions de têtes en 2015 et s'inscrit à la baisse, est assez concentré géographiquement (Roguet et al 2015). La France arrive au premier rang (22\% du cheptel communautaire) devant l'Allemagne (14\%), le Royaume-Uni (11\%), l'Irlande (7\%), l'Italie (7\%), la Pologne qui s'élève à 7,8 millions de tec. La consommation individuelle de viande bovine des européens (15 kg par an en 2015), qui est plus de deux fois inférieure à celle des deux pays précités, varie beaucoup d'un État membre à l'autre (exemple : 23,6 kg en France, 13,6 kg en Allemagne, $3,8 \mathrm{~kg}$ en Roumanie, $1,5 \mathrm{~kg}$ en Pologne).

Dans le secteur bovin (animaux vivants et viande bovine), les échanges de l'UE avec les pays tiers sont globalement limités. En effet, les exportations du secteur bovin (1,64 milliard d'euros en 2015) représentent seulement $1,3 \%$ de l'ensemble des exportations agroalimentaires de l'UE; de même, les importations de ce secteur (2,02 milliards d'euros en 2015) en représentent $1,8 \%$. Les exportations extra-UE dans le secteur bovin sont, par ailleurs, huit fois moins importantes (en valeur) que les flux intra-UE.

\section{1 / Le commerce extérieur de l'UE dans le secteur bovin}

En 2015, la balance commerciale de l'UE est excédentaire en bovins vivants (+ 776000 têtes pour un montant de 916 millions d'euros). Si les importations sont pratiquement nulles, à l'exception de quelques rares achats réalisés en Suisse et au Canada, les exportations à destination des pays du pourtour méditerranéen se développent, surtout depuis 2010 avec la Turquie. La balance commerciale de l'UE en viande bovine est, en revanche, négative à cette même date (- 62000 tec pour - 1,3 milliard d'euros). Il en résulte une balance commerciale négative pour le secteur bovin européen pris dans sa globalité (- 384 millions d'euros en 2015), à un niveau cependant inférieur à la moyenne annuelle 2000-2015 (- 594 millions d'euros).

a) Les échanges extérieurs de l'UE en bovins vivants

Depuis 2000, les exportations de l'UE en bovins vivants se sont élevées, en moyenne annuelle, à 442000 têtes. Elles ont donc toujours représenté moins de $1 \%$ du cheptel communautaire. Les principaux États membres impliqués sont la France $(16 \%$ du total des exportations européennes, soit 71000 têtes par an sur la période 2000-2015), l'Allemagne (14\%), la Pologne (12\%), la Hongrie $(10 \%)$, la Roumanie $(8 \%)$ et l'Espagne (7\%). Les exportations européennes de bovins vivants ont fortement varié au cours de la période étudiée en fonction surtout de la situation sanitaire respective des principaux États membres fournisseurs, des politiques commerciales, des besoins intérieurs des pays clients et des rapports concurrentiels avec les pays du Mercosur ou d'Océanie. Ainsi, elles sont passées de 462000 têtes en 2000 
à 271000 têtes par an au cours de la période 2005-2007 pour remonter à 776000 têtes en 2015 (figure 8). Ces flux ont été perturbés par les deux crises de l'ESB (1996 et 2001) et plus récemment par l'épisode de la Fièvre Catarrhale Ovine (FranceAgriMer, 2011c). En 2015, ces exportations concernent des veaux (328 000 têtes, dont 75\% de veaux d'un poids supérieur à $160 \mathrm{~kg}$ ), des bovins reproducteurs (154 000 têtes, dont 95\% de génisses) et des gros bovins, hors reproducteurs (294 000 têtes, dont 70\% de bovins mâles de plus de $300 \mathrm{~kg}$ ).

En 2015, la Turquie occupe le premier rang des pays importateurs en provenance de l'UE, avec 261000 têtes (soit un tiers de l'ensemble des exportations de l'UE), dont 80900 têtes en provenance de la France, 63200 têtes de la Hongrie et 30000 têtes de la République Tchèque. Dans un marché intérieur stimulé par la croissance démographique (près de 80 millions d'habitants en 2016) et l'amélioration du pouvoir d'achat, notamment dans les grandes villes, ce pays connaît des difficultés pour développer une offre intérieure de viande bovine qui soit à la hauteur de ses besoins : la production bovine est dispersée dans des exploitations de petite taille et souvent peu spécialisées ; les productions fourragères sont peu productives ; le recours aux achats d'aliments, dans un contexte de prix volatils, pénalise la rentabilité des élevages ; la structuration de la filière d'aval est insuffisante. Les importations de la Turquie concernent surtout des animaux de 6 à 10 mois, de moins de $300 \mathrm{~kg}$, destinés à être engraissés pour ensuite être abattus sur place selon le rituel halal. Si ce marché, qui était très difficile d'accès pour l'UE avant 2010 en raison des droits de douane dissuasifs appliqués, s'est largement ouvert, il reste potentiellement fragile. Outre les questions sanitaires et géopolitiques, le transport d'animaux vivants sur longue distance est, de plus, clairement contesté par certains acteurs de la société civile soucieux du bien-être animal. Le Liban est la deuxième porte d'entrée des bovins vivants européens en 2015 , avec $20 \%$ du total des exportations. En dépit de sa petite taille (4,5 millions d'habitants, du moins sans intégrer l'arrivée récente massive de réfugiés syriens), ce pays est structurellement déficitaire en viande bovine. Le cheptel reproducteur étant particulièrement limité, les abattages de bovins réalisés au Liban sont issus à plus de $90 \%$ d'animaux importés de l'UE, mais aussi de l'Uruguay et du Brésil. Ce pays est une destination traditionnelle pour l'UE, ses importations allant jusqu'à représenter $43 \%$ des exportations de l'UE au cours de la période 2000-2004. En moyenne 2000-2015, les principaux fournisseurs du Liban sont la France (28\% des exportations européen-
Figure 8. Les exportations de l'UE-28 en bovins vivants (nombre de têtes). (Source : Comext/Traitement INRA, SMART-LERECO).

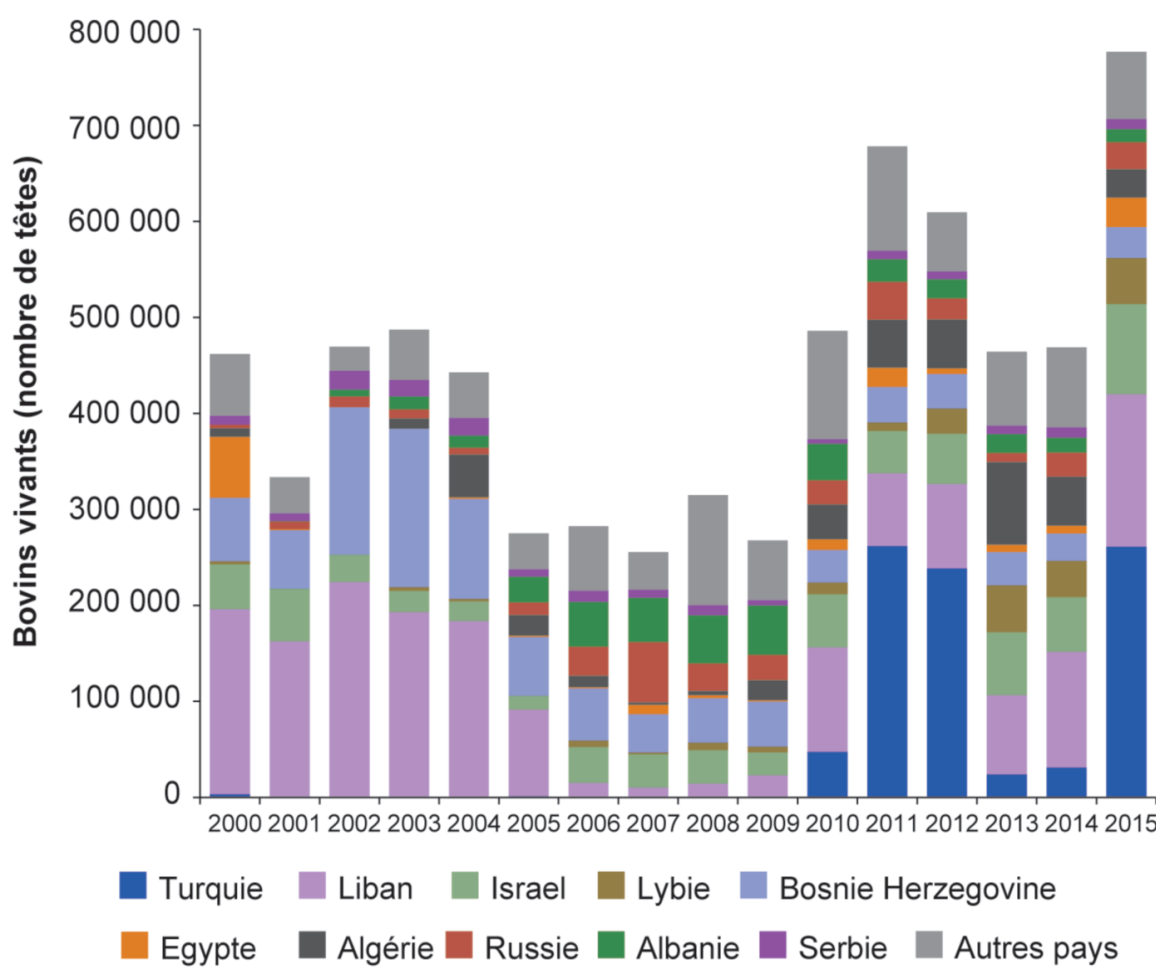

nes), l'Allemagne (28\%) et l'Espagne $(15 \%)$. La Slovénie et la Croatie occupent cependant une place grandissante. Avec huit millions d'habitants, Israël occupe la troisième position des pays clients de l'UE en bovins vivants (12\% des exportations de l'UE en 2015). Concurrencées par les expéditions de l'Australie, les exportations de l'UE vers ce pays n'ont cependant jamais excédé les 100000 têtes. En moyenne 2000-2015, les principaux pays fournisseurs d'Israël sont la Hongrie (29\%), la Lituanie $(29 \%)$, la Pologne $(17 \%)$ et la Roumanie $(15 \%)$. Les autres pays clients de l'UE en bovins vivants sont la Lybie (6\% des exportations de l'UE), la Bosnie Herzégovine (4\%), l'Égypte (4\%), la Tunisie (4\%), la Russie $(3 \%)$, l'Albanie $(2 \%)$ et la Tunisie (2\%).

b) Les échanges extérieurs de l'UE en viande bovine

Les importations de l'UE en viande bovine se sont élevées, en moyenne sur la période 2000-2015, à 385000 tec, soit environ $5 \%$ de la consommation intérieure. Les principaux pays importateurs sont le Royaume-Uni (29\% des importations européennes), les Pays-Bas (18\%), l'Allemagne (15\%) et l'Italie $(15 \%)$. Avec seulement $2 \%$ des importations européennes, la France est nettement en retrait. Les importations d'un État membre en provenance du marché mondial, comme c'est souvent le cas aux Pays-Bas, ne sont pas nécessairement destinées à fournir son propre marché intérieur. Les ports de Rotterdam (1 $\mathrm{er}$ port européen), d'Anvers (2ème) ou de Hambourg (3 ème) jouent, en effet, un rôle important dans l'importation de marchandises destinées, in fine, plus au marché européen qu'au pays importateur stricto sensu. Les importations de l'UE en viande bovine ont atteint un pic en 2006 (552000 tec); elles ont baissé à seulement 290000 tec en 2012 pour remonter à 310000 tec en 2015 (dont $66 \%$ de viande réfrigérée désossée, $21 \%$ de viande congelée et $12 \%$ de viande transformée).

Le Brésil est de loin le premier pays fournisseur de l'UE en viande bovine, avec $53 \%$ du total des importations de la période 2000-2015 (figure 9). Les importations européennes en provenance du Brésil, qui arrivent à $33 \%$ au RoyaumeUni et $18 \%$ en Italie, ont atteint un pic en 2006 (396 000 tec). La modification des règles sanitaires imposées par l'UE (cf. section 1.3) et la baisse des besoins européens en viande bovine ont ensuite entrainé une réduction drastique des importations, lesquelles s'établissent à 131000 tec en 2015. L'Argentine est le deuxième pays fournisseur de l'UE, avec $19 \%$ des achats européens de la période 2000-2015. Les importations européennes en provenance de ce pays ont, elles aussi, baissé en passant de 117000 tec en 2009 (année du pic) à seulement 42000 tec en 2015. Si les exportateurs argentins ont, dans un premier temps, profité du repli des exportations brésiliennes à partir de 2008, ils ont ensuite été confrontés à des difficultés liées à 
Figure 9. Les importations de l'UE-28 en viande bovine (Milliers de tec). (Source : Comext/Traitement INRA, SMART-LERECO).

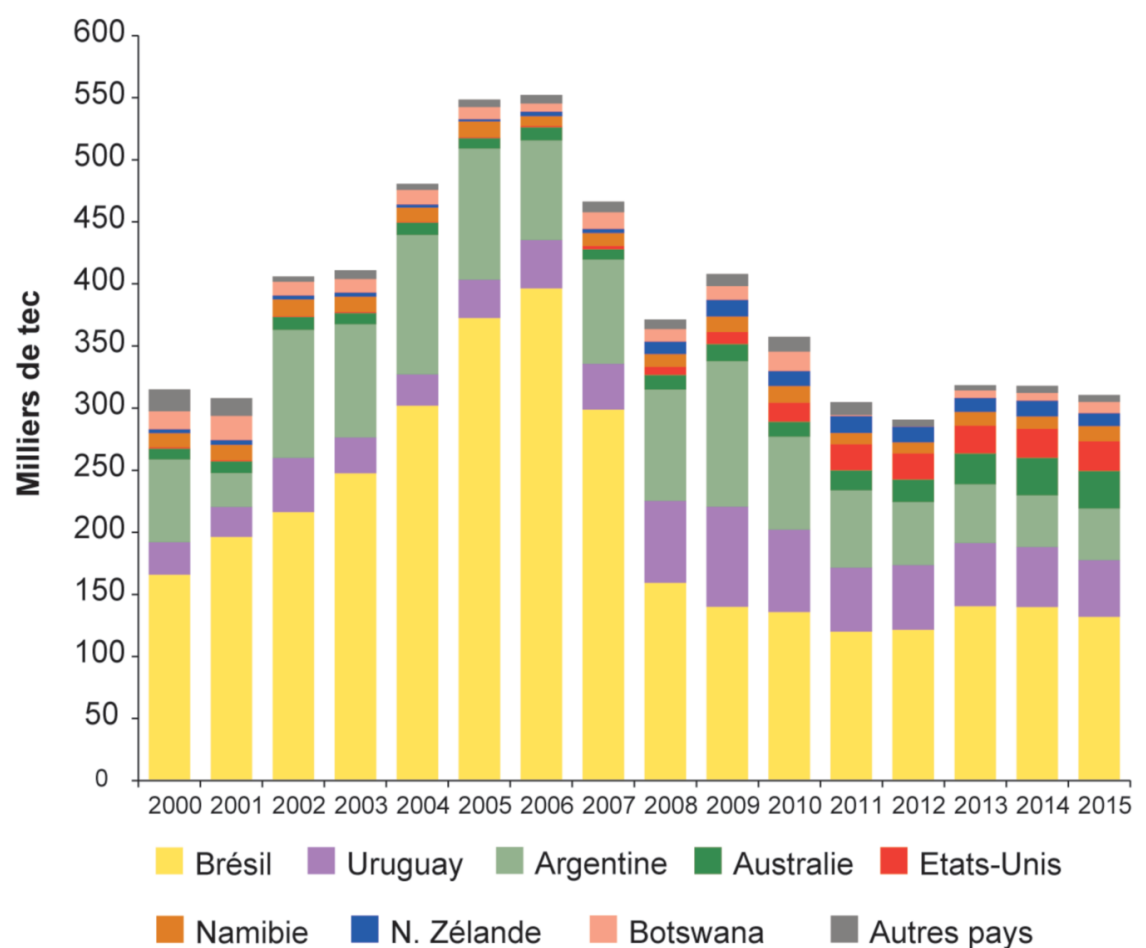

Figure 10. Les exportations de l'UE-28 en viande bovine (Milliers de tec). (Source : Comext/Traitement INRA, SMART-LERECO).

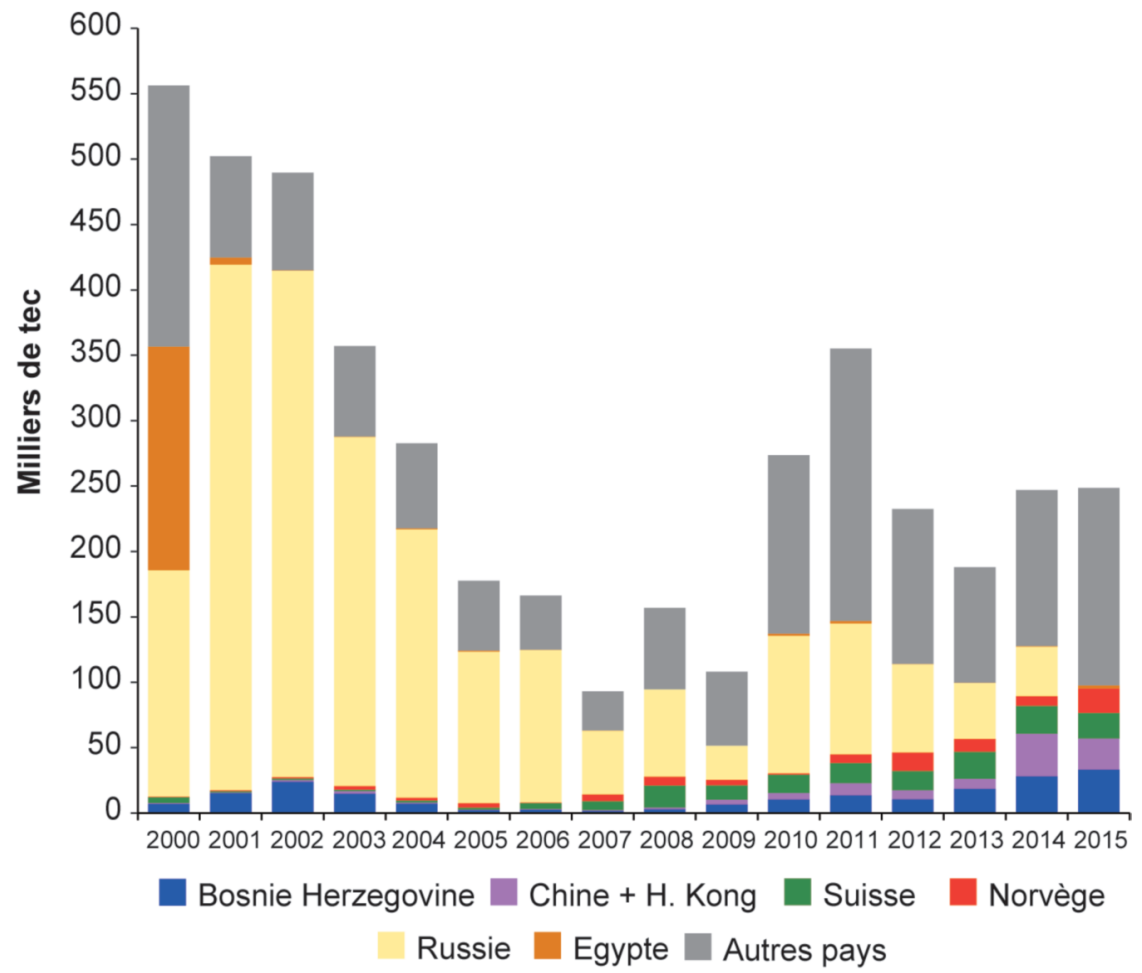

l'adoption par le gouvernement argentin de taxes à l'exportation (pour éviter une inflation trop forte sur les prix intérieurs de la viande bovine), au recul des surfaces de prairies sous la pression du développement des cultures (dont le soja qui bénéficiait d'une remontée des prix) et à une baisse des investissements. Le troisième pays fournisseur de l'UE, l'Uruguay,

également membre du Mercos (12\% des importations européennes de l'offre. Les autres pays fournisseurs de l'UE en viande bovine sont, par ordre décroissant d'importance, l'Australie $(3,8 \%)$, la Namibie $(2,9 \%)$, le Botswana $(2,6 \%)$, les États-Unis $(2,4 \%)$ et la Nouvelle-Zélande $(1,9 \%)$.

Les exportations de l'UE en viande bovine se sont élevées, en moyenne 2000-2015, à 277000 tec, soit $4 \%$ de la production de viande bovine. Les principaux pays exportateurs sont l'Allemagne (24\% des exportations), l'Irlande (17\%), la Pologne (11\%) et l'Italie (10\%). Avec $6 \%$ des exportations européennes, la France est, ici aussi, en retrait. Comme pour les importations, les exportations ont également fortement fluctué au cours de la période étudiée, passant de 556000 tec en 2000 à seulement 93000 tec en 2007 pour remonter à 248000 tec en 2015 (pour 724 millions d'euros). Sous la pression des règles de l'OMC, l'abandon des restitutions aux exportations a évidemment fragilisé la présence de la viande bovine européenne sur le marché international. En 2015, ces exportations relèvent pour $51 \%$ de viande congelée, $38 \%$ de viande réfrigérée et $11 \%$ de viande congelée.

La hiérarchie des pays clients de l'UE a évolué au fil de temps (figure 10). Si, au début des années 2000, la Russie était le premier client de l'UE (avec 401000 tec d'importations en 2001), ses achats ont considérablement baissé dès 2005 (116 000 tec). Ils ne sont jamais repartis à la hausse depuis et ils ont été nuls en 2015 (année d'application de l'embargo). Les importations de la Russie se font désormais surtout avec les pays du Mercosur, lesquels ont connu parallèlement une baisse de leurs débouchés avec le recul des importations européennes. L'Égypte, qui avait importé 170000 tec de viande bovine depuis l'UE en 2000, s'est ensuite orientée vers d'autres fournisseurs, suite à l'arrêt des restitutions européennes. En 2015, les pays destinataires de la viande bovine européenne sont nombreux mais pour des volumes faibles. Ainsi, les produits européens occupent une place souvent marginale dans l'approvisionnement de certains de ces pays. Avec $13 \%$ des exportations européennes de viande bovine, la Bosnie Herzégovine occupe la première place des pays destinataires devant Hong-Kong $(10 \%)$, la Suisse $(8 \%)$, la Norvège $(7 \%)$, la Macédoine (4\%), la Turquie (4\%), les Philippines (3\%), le Vietnam (3\%), 1'Angola (3\%), la Serbie (3\%) et le Ghana (3\%). 2000-2015). Comme en Argentine, les exportations de l'Uruguay à destination de l'UE ont d'abord progressé pour atteindre leur maximum en 2009 (81 000 tec), avant de redescendre à 45000 tec en 2015 ; la décapitalisation du cheptel allaitant ayant contribué à une limitation

\section{2 / Le commerce intra-UE dans le secteur bovin}

Les échanges de bovins-vivants entre les États membres européens (3,8 millions de têtes par an en moyenne sur la période 
2000-2015) sont huit fois plus élevés que les flux extra-UE (442 000 têtes). Les principaux déterminants de ces flux intra-communautaires sont la variabilité des taux d'auto-approvisionnement, l'hétérogénéité des préférences des consommateurs, les prix et la proximité géographique.

Les principaux pays européens qui exportent des bovins vivants à destination d'un autre État membre de l'UE sont la France (35\% des flux totaux), l'Allemagne (17\%) et la Pologne (11\%). Les exportations de la France en bovins vivants à destination de l'UE $(1,34$ million de têtes en moyenne annuelle 2000-2015) se font essentiellement vers l'Italie $(68 \%)$ et l'Espagne $(25 \%)$. Après avoir atteint 1,65 million de têtes en 2003 , elles ont baissé de près de $30 \%$ pour s'établir à 1,17 million de têtes en 2015 (tableau 1). Si la France demeure le premier fournisseur de l'Italie en bovins vivants (surtout des broutards destinés à l'engraissement), en représentant $77 \%$ de leurs achats en 2015 , le marché italien devient plus difficile en raison de la baisse de la consommation intérieure de viande bovine (Institut de l'Élevage, 2017). L'Italie reste cependant un débouché déterminant pour les veaux mâles issus du cheptel allaitant français, ceuxci étant engraissés pour une part importante dans la plaine du Pô. Les exportations françaises de bovins vivants s'orientent aussi, mais dans une moindre mesure, vers l'Espagne (132 000 têtes en 2008 et un pic à 449000 têtes en 2013). Deuxième exportateur européen de bovins vivants, l'Allemagne oriente ses exportations surtout vers les Pays-Bas (62\% du total en moyenne 2000-2015). Ces flux concernent des types d'animaux différents par rapport au cas précédent, à savoir des veaux à l'engrais de moins de $80 \mathrm{~kg}$. Les exportations de la Pologne en bovins vivants concernent aussi des veaux à l'engrais destinés aux Pays-Bas et à l'Italie. Si les trois principaux importateurs de bovins vivants en provenance d'un pays partenaire sont l'Italie (32\%), l'Espagne (20\%) et les Pays-Bas (20\%), les animaux importés ne sont pas de même type.

Les échanges intra-UE de viande bovine ont augmenté en passant de 1,67 million de tec en moyenne 2000-2003 à 2,68 millions de tec en moyenne 2011-2015. En valeur, ils sont nettement plus élevés (11,1 milliards d'euros en 2015) que ceux de bovins vivants (2,3 milliards d'euros). Les principaux pays européens exportateurs de viande bovine en intra-UE sont les Pays-Bas (18\% des flux en volume sur la période 2000-2015, y compris des volumes importants de viande de veau à prix élevé, à destination surtout de l'Italie et de la France), l'Irlande (17\%), l'Allemagne (17\%), la France (10\%) et la Pologne $(10 \%)$. Les exportations néerlandaise, irlandaise ou allemande de viande bovine à destination de l'UE sont ainsi plus importantes en volume que l'ensemble des exportations de l'UE à destination des marchés internationaux (tableau 2). Les principaux pays importateurs de viande bovine en intra-UE sont l'Italie (17\% des flux européens sur la même période), la France (15\%), le Royaume-Uni (12\%), les Pays-Bas (12\%) et l'Allemagne (10\%).

$\mathrm{Au}$ final, il ressort de cette analyse sur les flux extra-UE et intra-UE que la moitié des États membres de l'UE ont,

Tableau 1. Les exportations des Etats membres de I'UE-28 en bovins vivants (en milliers de têtes). Classement des pays par ordre décroissant des exportations totales en 2015.

(Source : Comext/Traitement INRA, SMART-LERECO).

\begin{tabular}{|c|c|c|c|c|c|c|c|c|}
\hline & \multicolumn{4}{|c|}{ Intra-UE } & \multicolumn{4}{|c|}{ Extra-UE } \\
\hline & $2000-04$ & 2005-09 & $2010-14$ & 2015 & $2000-04$ & 2005-09 & $2010-14$ & 2015 \\
\hline France & 1530 & 1312 & 1228 & 1175 & 68 & 23 & 107 & 148 \\
\hline Pays-Bas & 92 & 104 & 343 & 860 & 7 & 15 & 16 & 14 \\
\hline Allemagne & 651 & 625 & 626 & 752 & 101 & 42 & 47 & 62 \\
\hline R. Tchèque & 60 & 140 & 172 & 200 & 5 & 2 & 8 & 34 \\
\hline Roumanie & 60 & 131 & 195 & 143 & 19 & 31 & 53 & 63 \\
\hline Espagne & 107 & 104 & 95 & 63 & 10 & 18 & 51 & 115 \\
\hline Irlande & 86 & 136 & 171 & 172 & 38 & 3 & 8 & 4 \\
\hline Pologne & 465 & 578 & 255 & 120 & 125 & 29 & 19 & 19 \\
\hline Hongrie & 91 & 76 & 48 & 34 & 23 & 23 & 82 & 104 \\
\hline Autriche & 110 & 113 & 112 & 104 & 5 & 9 & 24 & 21 \\
\hline Belgique & 232 & 176 & 134 & 120 & 0 & 0 & 0 & 0 \\
\hline Lituanie & 10 & 112 & 87 & 92 & 2 & 14 & 28 & 15 \\
\hline Slovaquie & 40 & 82 & 94 & 70 & 0 & 1 & 6 & 24 \\
\hline Slovénie & 0 & 17 & 33 & 34 & 3 & 4 & 24 & 44 \\
\hline Croatie & 0 & 0 & 1 & 9 & 0 & 8 & 27 & 58 \\
\hline Portugal & 2 & 32 & 43 & 51 & 0 & 0 & 0 & 10 \\
\hline Danemark & 51 & 11 & 44 & 53 & 2 & 2 & 3 & 4 \\
\hline Italie & 44 & 51 & 46 & 47 & 2 & 0 & 1 & 6 \\
\hline Estonie & 3 & 17 & 32 & 35 & 0 & 0 & 4 & 6 \\
\hline Luxembourg & 34 & 32 & 32 & 38 & 0 & 0 & 0 & 0 \\
\hline Lettonie & 0 & 22 & 37 & 28 & 0 & 0 & 3 & 8 \\
\hline Bulgarie & 0 & 4 & 1 & 1 & 27 & 52 & 25 & 15 \\
\hline Royaume-Uni & 0 & 10 & 10 & 6 & 0 & 0 & 0 & 0 \\
\hline Chypre & 0 & 0 & 0 & 0 & 1 & 1 & 1 & 3 \\
\hline Finlande & 0 & 1 & 0 & 0 & 0 & 0 & 1 & 0 \\
\hline Suède & 2 & 1 & 0 & 1 & 0 & 1 & 0 & 0 \\
\hline Grèce & 0 & 0 & 1 & 0 & 0 & 0 & 4 & 0 \\
\hline Malte & 0 & 0 & 0 & 0 & 0 & 0 & 0 & 0 \\
\hline UE-28 & 3673 & 3885 & 3842 & 4209 & 439 & 279 & 541 & 777 \\
\hline
\end{tabular}


Tableau 2. Les échanges des États membres de l'UE-28 en viande bovine en 2015 (milliers de tec).

Classement des pays par ordre décroissant du solde commercial.

(Source : Comext/Traitement INRA, SMART-LERECO).

\begin{tabular}{|c|c|c|c|c|c|c|c|c|c|}
\hline & \multicolumn{3}{|c|}{ Exportations } & \multicolumn{3}{|c|}{ Importations } & \multicolumn{3}{|c|}{ Solde } \\
\hline & $\begin{array}{l}\text { Intra- } \\
\text { UE }\end{array}$ & $\begin{array}{c}\text { Extra- } \\
\text { UE }\end{array}$ & Total & $\begin{array}{l}\text { Intra- } \\
\text { UE }\end{array}$ & $\begin{array}{c}\text { Extra- } \\
\text { UE }\end{array}$ & Total & $\begin{array}{c}\text { Intra- } \\
\text { UE }\end{array}$ & $\begin{array}{c}\text { Extra- } \\
\text { UE }\end{array}$ & Total \\
\hline Irlande & 472 & 18 & 490 & 32 & 0 & 32 & 441 & 18 & 459 \\
\hline Pologne & 371 & 51 & 423 & 27 & 0 & 27 & 345 & 51 & 396 \\
\hline Belgique & 167 & 7 & 174 & 66 & 12 & 78 & 101 & -5 & 96 \\
\hline Autriche & 118 & 15 & 133 & 49 & 0 & 49 & 69 & 15 & 84 \\
\hline Pays-Bas & 478 & 20 & 498 & 322 & 96 & 418 & 156 & -77 & 80 \\
\hline Espagne & 166 & 17 & 183 & 118 & 18 & 136 & 48 & -1 & 47 \\
\hline Lituanie & 30 & 3 & 34 & 2 & 0 & 2 & 28 & 3 & 31 \\
\hline Lettonie & 10 & 0 & 10 & 6 & 0 & 6 & 4 & 0 & 4 \\
\hline Estonie & 3 & 0 & 3 & 4 & 0 & 4 & 0 & 0 & 0 \\
\hline Hongrie & 13 & 1 & 13 & 15 & 0 & 15 & -2 & 1 & -1 \\
\hline Allemagne & 364 & 35 & 399 & 356 & 45 & 402 & 8 & -11 & -3 \\
\hline Chypre & 0 & 0 & 0 & 2 & 1 & 3 & -2 & -1 & -3 \\
\hline Luxembourg & 4 & 0 & 4 & 9 & 0 & 9 & -4 & 0 & -5 \\
\hline Slovénie & 3 & 3 & 6 & 12 & 0 & 12 & -9 & 3 & -6 \\
\hline Malte & 0 & 0 & 0 & 7 & 1 & 7 & -7 & -1 & -7 \\
\hline Croatie & 5 & 3 & 8 & 19 & 0 & 19 & -14 & 3 & -11 \\
\hline Bulgarie & 2 & 1 & 2 & 14 & 0 & 14 & -12 & 1 & -12 \\
\hline Slovaquie & 4 & 0 & 4 & 18 & 0 & 18 & -14 & 0 & -14 \\
\hline Finlande & 4 & 0 & 4 & 21 & 0 & 21 & -17 & 0 & -17 \\
\hline R. Tchèque & 11 & 0 & 12 & 36 & 0 & 36 & -24 & 0 & -24 \\
\hline Roumanie & 8 & 0 & 8 & 34 & 0 & 34 & -26 & 0 & -26 \\
\hline Danemark & 82 & 7 & 90 & 122 & 2 & 124 & -40 & 6 & -34 \\
\hline Portugal & 9 & 1 & 11 & 109 & 2 & 111 & -100 & -1 & -101 \\
\hline Suède & 15 & 1 & 16 & 116 & 4 & 120 & -101 & -3 & -104 \\
\hline France & 222 & 17 & 239 & 341 & 5 & 346 & -120 & 12 & -108 \\
\hline Grèce & 1 & 0 & 1 & 115 & 1 & 116 & -114 & -1 & -115 \\
\hline Royaume-Uni & 123 & 25 & 148 & 339 & 61 & 400 & -216 & -36 & -252 \\
\hline Italie & 142 & 23 & 164 & 383 & 62 & 445 & -241 & -39 & -280 \\
\hline
\end{tabular}

en 2015, un solde commercial négatif (en valeur) dans le secteur bovin. Les cinq principaux pays déficitaires sont l'Italie (- 2,41 milliards d'euros), le RoyaumeUni (- 1,13 milliard d'euros), la Grèce (- 425 millions d'euros), la Suède $(-417$ millions d'euros) et le Portugal ( -347 millions d'euros). Dans une quinzaine de pays, notamment les nouveaux entrants, le solde du secteur bovin se situe pas très loin de l'équilibre. Les cinq principaux pays excédentaires sont l'Irlande (+2,01 milliards d'euros), la Pologne (+ 1,19 milliard d'euros), la France (+ 810 millions d'euros), les Pays-Bas (+ 719 millions d'euros) et la Belgique (+ 408 millions d'euros). L'Irlande et la Pologne commercialisent essentiellement de la viande bovine. La France et 1'Allemagne ont un excédent en bovins vivants mais un déficit en viande bovine.

\section{Conclusion}

Le secteur bovin mondial bénéficie donc encore d'une croissance de la demande, surtout dans les pays asiatiques où le niveau individuel de consommation reste très largement inférieur à celui des pays développés. L'essor des échanges mondiaux de viande bovine s'accompagne de l'arrivée de deux nouveaux acteurs sur le marché, à savoir l'Inde pour les exportations et la Chine pour les importations. Moyennant des modèles productifs différents, l'Australie, les pays du Mercosur et les États-Unis occupent toujours une place centrale dans les exportations du secteur bovin. Leurs exportations ont cependant fortement fluctué au fil des quinze dernières années sous l'influence de leur situation sanitaire, des politiques commerciales adoptées par les pays importateurs, des parités monétaires, des coûts de production relatifs entre pays (l'augmentation du prix des céréales ayant eu un impact négatif sur les systèmes les plus intensifs) et de la dynamique de leur production bovine. En Chine, le développement de la production se heurte à des difficultés structurelles qui ne lui permettent pas de satisfaire intégralement et en temps réel les besoins intérieurs de viande bovine, ceci justifiant des achats massifs, y compris en viande indienne à bas prix transitant illégalement par le Vietnam. Les ÉtatsUnis, où la production de viande bovine a légèrement baissé, présentent une situation singulière en étant tout à la fois un grand pays exportateur et importateur de viande bovine. Ses approvisionnements en provenance surtout de l'Océanie et des partenaires de l'ALENA permettent de satisfaire une demande intérieure soutenue, comme c'est également le cas dans les pays du Mercosur. Si le Japon et la Russie demeurent des grands acheteurs de viande bovine, les flux d'importations sont désormais décroissants. L'UE, qui fait face à une baisse de sa production et de sa consommation, n'est plus un acteur majeur du commerce international dans le secteur bovin, d'autant que son principal partenaire historique à l'export, la Russie, a modifié ses sources d'approvisionnement. La consommation de viande bovine des européens, plus de deux fois inférieure par habitant à celle des américains, est surtout satisfaite par une offre de viande bovine européenne et issue pour une large part du secteur laitier (et non pas allaitant comme la plupart des 
autres pays). Si les exportations extraUE de viande bovine reculent, les flux intra-UE se développent.

Même si la demande mondiale de viande bovine est croissante et que de nombreux pays ne seront pas en capacité de développer leur production à la hauteur de leurs besoins, il reste difficile de prévoir, avec précision, ce que seront les échanges internationaux dans le secteur bovin à horizon de dix ou vingt ans. La capacité des États impliqués à se prémunir des maladies qui affectent le secteur constitue un enjeu crucial et régulièrement mis en avant par les experts de l'OIE. La stratégie qui sera mise en œuvre par les, gouvernements, dont aujourd'hui les États-Unis tentés par plus de protectionnisme, pour s'engager ou non dans des accords commerciaux bilatéraux ou multilatéraux aura également son importance car les droits de douane appliqués aux frontières diffèrent souvent d'un pays à l'autre. Les débats sociétaux qui ont trait aux effets environnementaux de la production bovine (production de gaz à effet de serre, faible efficacité de transformation des végétaux en protéines animales...), au bien-être animal (conditions d'abattage des bovins et mode de transport sur longue distance...) et à la santé humaine (liens entre la consommation de la viande bovine et le développement de certaines maladies) ne doivent pas, non plus, être négligés dans une perspective à long terme.

\section{Remerciements}

L'auteur remercie Cécile Le Roy (INRA, SMART-LERECO, Nantes) pour son appui dans le traitement des bases de données BACI et COMEXT. Il remercie aussi les experts du département Économie de l'Institut de l'Elevage, notamment Philippe Chotteau, pour la qualité des informations régulièrement publiées sur ce vaste thème et pour les échanges que nous avons.

\section{Références}

ABIEC, 2016. Brazilian livestock profile. Annual report, $46 \mathrm{p}$.

Bell A.W., Charmley E., Hunter R.A., Archer J.A., 2011. The Australasian beef industries: challenges and opportunities in the $21^{\text {st }}$ century. Anim. Frontiers, 22, 10-19.

Bureau J.C., 2013. The US Farm Bill: lessons for CAP reform? Int. J. Agricult. Manag., 2, 67-69.

Champion F., Chotteau P., 2013. Compétitivité de la filière viande bovine brésilienne et place sur le marché mondial. Renc. Rech. Rum., 4p.

Chatellier V., 2016. Le commerce international, européen et français de produits laitiers : évolutions tendancielles et dynamiques concurrentielles. INRA Prod. Anim., 29, 143-162.

Chaumet J.M., Pouch T., 2017. La Chine au risque de la dépendance alimentaire. Éditions Presse Universitaire de Rennes, France, 212p.

Commission européenne, 2016. Agri-food trade in 2015: China boosts EU exports. Monitoring agri-trade policy, 1, 24p

Cordonnier C., Hervé J.J., 2003. Agriculture russe : les paradoxes du renouveau. Le Demeter 2004, Editions Armand Colin, 7-66.

Edwards B., Waldron S., Brown C., Longworth J., 2016. The Sino-Australian cattle and beef relationship: assessment and prospects. Report of the Australia-China Relations Institute, 99p.

FAO, 2016. Perspectives de l'alimentation : les marchés en bref. Rapport, 12p.

FAO-OCDE, 2016. Perspectives agricoles 20162025. Rapport annuel, 144p.

FranceAgriMer, 2011a. La consommation mondiale de viande : état des lieux, dynamique et défis. Les synthèses, $5,8 \mathrm{p}$.

FranceAgriMer, 2011b. Les Global Players dans les filières viandes : américains et brésiliens aux premières places. Les synthèses, $9,12 \mathrm{p}$.

FranceAgriMer, 2011c. Les exportations françaises de bovins vivants vers les pays du pourtour méditerranéen. Les synthèses, 10, 7p.
FranceAgriMer, 2012. Le commerce international de la viande bovine : vers une stabilisation des échanges ? Les synthèses, 16, 15p.

Galyean M.L., Ponce C., Schutz J., 2011. The future of beef production in North America. Anim. Frontiers, 1, 29-36.

Gotoh T., Takahashi H., Nishimura T., Kuchida K., Mannen H., 2014. Meat produced by Japanese black cattle and Wagyu. Anim. Frontiers, 4, 46-54.

Greene J.L., 2015. Country of origin labeling for foods and the WTO trade dispute on meat labeling. Congres. Res. Service report 7-5700, $56 \mathrm{p}$.

Guanghong Z., Wangang Z., Xinglian X., 2012. China's meat industry revolution: challenges and opportunities for the future. Meat Sci., 92, 188-196.

Han X.P., Hubbert B., Hubbert M.E., Reinhardt C.D., 2016. Overview of the beef cattle industry in China: The widening deficit between demand and output in a vicious circle. J. Fisheries Livest. Prod., 4, 6p.

Henchion M., McCarthy M., Resconi V.C., Toy D., 2014. Meat consumption: trends and quality matters. Meat Sci., 98, 561-568.

Hogan L., Morris P., 2010. Agricultural and food policy choices in Australia. ABARE project 43014, 27p.

Huang Y., Hocquette J.F., Porry J.L., Chaumet J.M., Huo Y., 2015. Production de viande bovine en Chine et perspectives d'évolution. INRA Prod. Anim., 28, 259-270.

Institut de l'Élevage, 2012. La production de viande bovine au Brésil. Dossier Économie de l'Élevage, 427, 54p.

Institut de l'Élevage, 2015a. La filière viande bovine aux États-Unis : performante et offensive. Dossier Économie de l'Élevage, 459, 31p.

Institut de l'Élevage, 2015b. L'Inde, leader émergent sur le marché mondial de la viande bovine. Dossier Économie de l'Élevage, 463, 31p.

Institut de l'Élevage, 2016. Le marché mondial de la viande bovine : dans la tourmente de l'économie globale. Dossier Economie de l'Élevage, $468,38 \mathrm{p}$
Institut de l'Élevage, 2017. Bovins viande : conjonction de crises en 2016 et incertitudes géopolitiques en 2017. Dossier Économie de 1'Élevage, 475, 41p.

Jamet J.P., Chaumet J.M., 2016. Soybean in China: adaptating to the liberalization. OCL, 23, 9p.

Kahn L., Cottle D., 2014. Beef cattle production and trade. Csiro Publishing, 584p.

Landes M., Melton A., Edwards S., 2016. From where the Buffalo roam: India's beef exports. USDA, Report from the Economic Research Service, June, 34p.

Legrand I., Hocquette J.F., Polkinghorne R.J., Pethick D.W., 2013. Prediction of beef eating in France using the Meat Standards Australia system. Animal, 7, 524-529.

Liu D., Iqbal B.A., 2016. Sino-Indian non-normal bovine meat trade. Foreign Trade Review, 51, 98-107.

McEldowney J., 2016. The Russian ban on agricultural products. Europ. Parl. Res. Service, 581$971,12 \mathrm{p}$.

OCDE, 2009. Evaluation of agricultural policy reforms in Japan. Report, $121 \mathrm{p}$.

OMC, 2015. États-Unis : certaines prescriptions en matière d'étiquetage indiquant le pays d'origine (EPO). Décision de 1'arbitre WT/DS384/ $\mathrm{ARB}, 67 \mathrm{p}$.

Poncet R., Hofferer S., 2016. Opportunité du marché japonais pour l'agroalimentaire français. Note du service économique de l'Ambassade de France au Japon, 38p.

Potard-Hay G., 2016. La gestion publique des questions agricoles en Australie. Analyse du Centre d'Études et de Prospective, 91, 4p.

Pouch T., Kheraief N., 2016. Le commerce extérieur agroalimentaire de l'Inde. Économie Rurale, 352, 67-80.

Prikhodko D., Davleyev A., 2014. Russian Federation meat sector review. FAO report, 199p. Roguet C., Gaigné C., Chatellier V., Cariou S., Carlier M., Chenut R., Daniel K., Perrot C., 2015. Spécialisation territoriale et concentration 
des productions animales européennes : État des lieux et facteurs explicatifs. INRA Prod. Anim., $28,5-22$.

Sabourin, 2014. L'agriculture brésilienne en débat : évolutions récentes, controverses et politiques publiques. Problèmes d'Amérique latine, $95,33-55$.

Silva L.S., 2014. Export trends and free trade in Australia: an analysis. Otemon J. Austral. Stud., 40, 47-62.
USDA, 2011. The diverse structure and organization of US beef cow-calf farms. Econ. Inf. Bul., 73, 42p.

USDA, 2015a. Australia: livestock and products. Gain report, AS1524, 18p.

USDA, 2015b. India: livestock and products. Gain report, IN5106, 10p.

USDA, 2016. Japan: livestock and products. Gain report, JA6003, 40p.
USDA, 2017a. Livestock, dairy and poultry outlook. Economic Research Service report, M-272, 23p.

USDA, 2017b. China: livestock and products. Gain report, $\mathrm{CH} 17005,18 \mathrm{p}$.

USDA, 2017-c. Russia: livestock and products. Gain report, RS 1713, 29p.

Zhou G., Zhang W., Xu X., 2012. China's meat industry revolution: challenges and opportunities for the future. Meat Sci., 192, 188-196.

\section{Résumé}

L'augmentation de la consommation de viande bovine dans plusieurs pays asiatiques contribue à renforcer les jeux concurrentiels entre les principaux exportateurs mondiaux de viande bovine, dont l'Australie, l'Inde, le Brésil ou les États-Unis. Les principaux importateurs de viande bovine, dont les États-Unis, la Chine (avec Hong-Kong), le Japon et la Russie, connaissent des trajectoires différenciées en fonction de l'évolution de la demande intérieure en viande bovine, des conditions sanitaires propres aux pays fournisseurs et, parfois, de questions géopolitiques. L'Union Européenne, qui connait à la fois une baisse de sa production et de sa consommation de viande bovine, n'est pas un acteur majeur du commerce international dans ce secteur. La demande intérieure est satisfaite essentiellement par des produits européens, mais des flux importants de bovins vivants et de viande bovine ont lieu entre les États membres. En utilisant les bases de données des douanes disponibles, tant à l'échelle mondiale («Comtrade » et «Baci») que de l'Union Européenne ( Comext»), cet article propose une analyse sur l'évolution de la situation productive et commerciale des principaux acteurs du secteur bovin mondial pour la période 2000 à 2015.

\section{Abstract \\ Trade in live cattle and beef meat at world and EU levels: productive and commercial trajectories for the main countries involved}

The increase in beef consumption in several Asian countries is helping to strengthen competitive games between the world's leading beef exporters, including Australia, India, Brazil and the United States. The main importers of beef and veal, including the United States, China (with Hong-Kong), Japan and Russia, have different trajectories that depend on changes in domestic demand for beef and veal, sanitary conditions in supplier countries and sometimes geopolitical issues. The European Union, which is experiencing both a decline in its production and consumption of beef, is not a major player in international trade in this sector. Domestic demand is largely satisfied by European products, but significant flows of live cattle and beef take place between Member States. Using the available customs databases both on a global scale (Comtrade and Baci) and the European Union (Comext), this article proposes an analysis on the evolution of the productive and commercial situation of the main players in the beef cattle sector for the period 2000 to 2015.

CHATELLIER V., 2017. Les échanges de bovins vivants et de viande bovine dans le monde et dans l'UE : trajectoires productives et commerciales des principaux pays impliqués. INRA Prod. Anim., 30, 199-218. 
